FUNCTION SPACES XII

BANACH CENTER PUBLICATIONS, VOLUME 119

INSTITUTE OF MATHEMATICS

POLISH ACADEMY OF SCIENCES

WARSZAWA 2019

\title{
HARDY SPACES OF GENERAL DIRICHLET SERIES A SURVEY
}

\author{
ANDREAS DEFANT and INGO SCHOOLMANN \\ Institute of Mathematics, Carl von Ossietzky University Oldenburg \\ 26111 Oldenburg, Germany \\ E-mail:defant@mathematik.uni-oldenburg.de, ingo.schoolmann@uni-oldenburg.de
}

\begin{abstract}
The main purpose of this article is to survey on some key elements of a recent $\mathcal{H}_{p}$-theory of general Dirichlet series $\sum a_{n} e^{-\lambda_{n} s}$, which was mainly inspired by the work of Bayart and Helson on ordinary Dirichlet series $\sum a_{n} n^{-s}$. In view of an ingenious identification of Bohr, the $\mathcal{H}_{p}$-theory of ordinary Dirichlet series can be seen as a sub-theory of Fourier analysis on the infinite dimensional torus $\mathbb{T}^{\infty}$. Extending these ideas, the $\mathcal{H}_{p}$-theory of $\lambda$-Dirichlet series is built as a sub-theory of Fourier analysis on so-called $\lambda$-Dirichlet groups. A number of problems are added.
\end{abstract}

1. Introduction. Within the last two decades the theory of ordinary Dirichlet series $\sum a_{n} n^{-s}$ saw a sort of renaissance. The study of these series in fact was one of the hot topics in mathematics at the beginning of the 20th century. Among others, H. Bohr, Besicovitch, Bohnenblust, Hardy, Hille, Landau, Perron, M. Riesz, and Neder, were the leading mathematicians in this issue. The theory lived a sort of golden moment between the 1910's and the 1930's but after that it was somehow forgotten. Some 20 years ago the seminal article 37. of Hedenmalm, Lindqvist and Seip called again the attention from analysis to Dirichlet series. Since then a lot has been going on, and ordinary Dirichlet series have been studied with new techniques from functional and harmonic analysis.

Bohr's main interest was to derive properties of Dirichlet series from the analytical properties of the holomorphic functions defined by them. It is well known that Dirichlet series converge on halfplanes, and where they converge, they define a holomorphic function. For each Dirichlet series $D=\sum a_{n} n^{-s}$ Bohr considered three abscissas: $\sigma_{c}(D)$, $\sigma_{u}(D)$, and $\sigma_{a}(D)$ that define the maximal halfplanes on which the series converges,

2010 Mathematics Subject Classification: Primary 43A17; Secondary 30H10, 30B50.

Key words and phrases: general Dirichlet series, Hardy spaces.

The paper is in final form and no version of it will be published elsewhere. 
converges uniformly, or converges absolutely, respectively. He also considered a fourth abscissa, $\sigma_{b}(D)$, that gives the maximal halfplane on which the series converges and defines a bounded and holomorphic function. Then

$$
\sigma_{c}(D) \leq \sigma_{b}(D) \leq \sigma_{u}(D) \leq \sigma_{a}(D) .
$$

In 9] Bohr was interested on describing the absolute convergence abscissa of an ordinary Dirichlet series in terms of analytic properties of its limit function. He proved

$$
\sigma_{b}(D)=\sigma_{u}(D)
$$

(see e.g. [23, Corollary 1.14] or [45, Theorem 6.2.3, p. 145]), and then considered the number

$$
S=\sup \left\{\sigma_{a}(D)-\sigma_{u}(D): D=\sum a_{n} n^{-s}\right\},
$$

that gives the maximal width of the band on which a Dirichlet series can converge uniformly but not absolutely. Bohr showed that $S \leq 1 / 2$. The problem of whether or not this was the correct value remained open for some 15 years, until Bohnenblust and Hille in [7] indeed proved that

$$
S=\frac{1}{2}
$$

(see also [23, Theorem 4.1]). These ideas are the seeds of a recent revival of interest in the research area opened up by these early contributions. A new field emerged intertwining the classical work in novel ways with modern functional analysis, infinite dimensional holomorphy, probability theory as well as analytic number theory. As a consequence, a number of challenging research problems crystallized and were solved over the last decades. We refer to the monographs [40], [45], and [23] where many of the key elements of these new developments for ordinary Dirichlet series are described in detail.

A fundamental object in these investigations is given by the Banach space $\mathcal{D}_{\infty}$ of all ordinary Dirichlet series $D=\sum a_{n} n^{-s}$ which converge and define a bounded, and then necessarily holomorphic, function on $[R e>0]$ (endowed with the supremum norm on $[R e>0])$. Let us mention that the established notation for this space is $\mathcal{H}_{\infty}$; but we here prefer to change the notation slightly since we later in the framework of general Dirichlet series invent the spaces $\mathcal{D}_{\infty}(\lambda)$ and $\mathcal{H}_{\infty}(\lambda)$ which in general differ.

A celebrated result from [37] shows that $\mathcal{D}_{\infty}$ in fact equals the Hardy space $H_{\infty}\left(\mathbb{T}^{\infty}\right)$ on the infinitely dimensional torus. Let us explain this in more detail. The infinitely dimensional torus $\mathbb{T}^{\infty}$ is the infinite product of $\mathbb{T}=\{w \in \mathbb{C}:|w|=1\}$ which forms a natural compact abelian group on which the Haar measure is given by the normalized Lebesgue measure. The characters on $\mathbb{T}^{\infty}$, so the elements in the dual group, consist of all monomials $z \mapsto z^{\alpha}$, where $\alpha=\left(\alpha_{k}\right) \in \mathbb{Z}^{(\mathbb{N})}$ (all finite sequences of integers), and $H_{\infty}\left(\mathbb{T}^{\infty}\right)$ denotes the closed subspace of all $f \in L_{\infty}\left(\mathbb{T}^{\infty}\right)$ such that the Fourier coefficient

$$
\widehat{f}(\alpha)=\int_{\mathbb{T}^{\infty}} f(w) w^{-\alpha} d w=0,
$$

whenever $\alpha<0$ (in the sense that some $\alpha_{k}<0$ ). Then, based on Bohr's work, it is proven in [37] (see also [23, Corollary 5.3] or [45, Proposition 6.5.3, p. 159]) that there is 
a unique linear isometry

$$
H_{\infty}\left(\mathbb{T}^{\infty}\right)=\mathcal{D}_{\infty}
$$

which preserves Fourier and Dirichlet coefficients in the sense that

$$
\widehat{f}(\alpha)=a_{n} \text { whenever } n=\mathfrak{p}^{\alpha}:=p_{1}^{\alpha_{1}} \ldots p_{N}^{\alpha_{N}},
$$

where $\alpha=\left(\alpha_{1}, \ldots, \alpha_{N}, 0, \ldots\right) \in \mathbb{N}_{0}^{(\mathbb{N})}$ and $\mathfrak{p}=2,3,5, \ldots$ the sequence of primes. We refer to this result as the Bohr-Hedenmalm-Lindqvist-Seip theorem (see also [23, Corollary 5.3]). The crucial point of its proof is the fact that each natural number has a unique prime number decomposition, as well as Kronecker's theorem on Diophantine approximation (see e.g. [23, Proposition 3.4]): The continuous group homomorphism

$$
\beta: \mathbb{R} \rightarrow \mathbb{T}^{\infty}, \quad t \mapsto\left(p_{k}^{-i t}\right)_{k=1}^{\infty}
$$

has dense range, which in particular implies that for each $n$ and $\alpha$ with $n=\mathfrak{p}^{\alpha}$ the following diagram commutes:

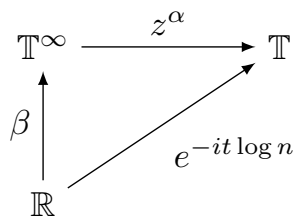

The original proof of (3) goes a detour through infinite dimensional holomorphy using a result of Cole and Gamelin from 21. Denote by $H_{\infty}\left(B_{c_{0}}\right)$ all holomorphic (Fréchet differentiable) and bounded functions $f$ on the open unit ball $B_{c_{0}}$ of $c_{0}$, which endowed with the sup norm forms a Banach space (as usual $c_{0}$ denotes the Banach space of all null sequences). Then Cole and Gamelin show that there exists a unique isometric linear bijection

$$
H_{\infty}\left(\mathbb{T}^{\infty}\right)=H_{\infty}\left(B_{c_{0}}\right), f \mapsto g,
$$

which preserves Fourier and monomial coefficients in the sense that

$$
\widehat{f}(\alpha)=\frac{\partial^{\alpha} g(0)}{\alpha !},
$$

for every multi-index $\alpha$ (see [23, Theorem 5.1]).

The equality of Banach spaces from (3) shows that a Dirichlet series $D$ belongs to $\mathcal{D}_{\infty}$ if and only if there is a function $f \in H_{\infty}\left(\mathbb{T}^{\infty}\right)$ such that the Dirichlet coefficients $\left(a_{n}(D)\right)$ and the Fourier coefficients $(\widehat{f}(\alpha))$ coincide in the sense of (4), if and only if there is a function $g \in H_{\infty}\left(B_{c_{0}}\right)$ such that the Dirichlet coefficients $\left(a_{n}(D)\right)$ and the monomial coefficients $\left(\frac{\partial^{\alpha} g(0)}{\alpha !}\right)$ coincide in the sense of $(7)$. This links the theory of ordinary Dirichlet, which generate bounded and holomorphic functions on the positive halfplane, intimately with Fourier analysis on the group $\mathbb{T}^{\infty}$ as well as infinite dimensional holomorphy on the open unit ball of $c_{0}$.

More generally, Bayart in [4] developed an $\mathcal{H}_{p}$-theory of Dirichlet series. Recall that the Hardy space $H_{p}\left(\mathbb{T}^{\infty}\right), 1 \leq p \leq \infty$, is the closed subspace of all $f \in L_{p}\left(\mathbb{T}^{\infty}\right)$ such that $\widehat{f}(\alpha)=0$ only if $\alpha<0$. Then the Banach spaces $\mathcal{H}_{p}$ of ordinary Dirichlet series by 
definition is the isometric image of $H_{p}\left(\mathbb{T}^{\infty}\right)$ under the identification from (4):

$$
\mathcal{H}_{p}=H_{p}\left(\mathbb{T}^{\infty}\right)
$$

and the Bohr-Hedenmalm-Lindqvist-Seip theorem from $(6)$ reads

$$
\mathcal{D}_{\infty}=\mathcal{H}_{\infty}=H_{\infty}\left(\mathbb{T}^{\infty}\right) .
$$

Again the Banach spaces $\mathcal{H}_{p}$ can be reformulated in terms of holomorphic functions in infinitely many variables. Instead of looking at holomorphic functions on $B_{c_{0}}$, we look at holomorphic functions on $\ell_{2} \cap B_{c_{0}}$, understood as an open subset of $\ell_{2}$. Then

$$
H_{p}\left(\ell_{2} \cap B_{c_{0}}\right)
$$

is the Banach space of all holomorphic functions $g: \ell_{2} \cap B_{c_{0}} \rightarrow \mathbb{C}$ for which

$$
\|g\|_{H_{p}\left(\ell_{2} \cap B_{c_{0}}\right)}=\sup _{n \in \mathbb{N}} \sup _{0<r<1}\left(\int_{\mathbb{T}^{n}}\left|g\left(r w_{1}, \ldots, r w_{n}, 0,0, \ldots\right)\right|^{p} d\left(w_{1}, \ldots, w_{n}\right)\right)^{1 / p}<\infty .
$$

It is proven in [5, Theorem 3.9] that for $1 \leq p<\infty$ there is a unique isometric identity

$$
H_{p}\left(\ell_{2} \cap B_{c_{0}}\right)=H_{p}\left(\mathbb{T}^{\infty}\right),
$$

identifying Fourier and monomial coefficients in the sense of (7) (for a detailed proof see also [23, Chapter 13]).

Let us now face general Dirichlet series - our main object of interest. Given a frequency $\lambda=\left(\lambda_{n}\right)$ (i.e. a nonnegative strictly increasing sequence of real numbers tending to $+\infty$ ), a $\lambda$-Dirichlet series is a formal sum

$$
\sum a_{n} e^{-\lambda_{n} s}
$$

with complex Dirichlet coefficients $\left(a_{n}\right)$ and a complex variable $s$. We denote by $\mathcal{D}(\lambda)$ the space of all formal $\lambda$-Dirichlet series.

We start recalling some basic facts. The natural domains of convergence of Dirichlet series are halfspaces (see [36, Theorems 1, 2, p. 3]), and as in the ordinary case the 'abscissas'

$$
\begin{aligned}
& \sigma_{c}(D)=\inf \{\sigma \in \mathbb{R} \mid D \text { converges on }[R e>\sigma]\}, \\
& \sigma_{a}(D)=\inf \{\sigma \in \mathbb{R} \mid D \text { converges absolutely on }[R e>\sigma]\}, \\
& \sigma_{u}(D)=\inf \{\sigma \in \mathbb{R} \mid D \text { converges uniformly on }[R e>\sigma]\},
\end{aligned}
$$

rule the convergence theory of general Dirichlet series. Again general Dirichlet series $D$ define holomorphic functions on $\left[R e>\sigma_{c}(D)\right]$, which relies on the fact that they converge uniformly on all compact subsets of $\left[R e>\sigma_{c}(D)\right]$ (see again [36, Theorem 2, p. 3] or [45, Lemma 4.1.1, p. 97]).

There are useful Bohr-Cahen formulas for the abscissas $\sigma_{c}$ and $\sigma_{a}$, that are, given $D=\sum a_{n} e^{-\lambda_{n} s}$

$$
\sigma_{c}(D) \leq \limsup _{N} \frac{\log \left(\left|\sum_{n=1}^{N} a_{n}\right|\right)}{\lambda_{N}} \text { and } \sigma_{a}(D) \leq \limsup _{N} \frac{\log \left(\sum_{n=1}^{N}\left|a_{n}\right|\right)}{\lambda_{N}},
$$

where in each case we have equality if the left hand side is nonnegative. See [36, Theorems 7,8, p. 8] for a proof. The formula for $\sigma_{u}$ (and its proof) extends from the ordinary 
case (in [23, Proposition 1.6] or [45, Theorem 4.2.1]) canonically to arbitrary $\lambda$ 's:

$$
\sigma_{u}(D) \leq \limsup _{N} \frac{\log \left(\sup _{t \in \mathbb{R}}\left|\sum_{n=1}^{N} a_{n} e^{-\lambda_{n} i t}\right|\right)}{\lambda_{N}},
$$

where again the equality holds if the left hand side is nonnegative. For an extension of (11) with an explicit proof see [48, Proposition 2.4].

We point out that making the jump from the ordinary case $\lambda=(\log n)$ to arbitrary frequencies reveals serious difficulties and that many of the above ideas fail for general Dirichlet series. Much of the ordinary theory relies on Bohr's theorem from (1), the fact that for each ordinary Dirichlet series the abscissa of uniform convergence and boundedness coincide. This phenomenon fails for general Dirichlet series. Further due to the prime number theorem each natural number $n$ has its prime number decomposition $n=\mathfrak{p}^{\alpha}$ and so the frequency $(\log n)$ can be written as a linear combination of $\left(\log p_{j}\right)$ with natural coefficients. This intimately links the theory of ordinary Dirichlet series with the theory of holomorphic functions on polydiscs, and in particular with the theory of polynomials $\sum c_{\alpha} z^{\alpha}$ in finitely many complex variables. One of several consequences is that $m$-homogeneous Dirichlet series $\sum a_{n} n^{-s}$, i.e. $a_{n} \neq 0$ only if $n$ has $m$ prime factors, are linked with $m$-homogeneous polynomials. This way powerful tools enter the game, as e.g. polynomial inequalities (like the Bohnenblust-Hille inequalities, hypercontractivity of convolution with the Poisson kernel, etc.), m-linear forms, or polarization. So modern Fourier analysis and infinite dimensional holomorphy enrich the theory of ordinary Dirichlet series considerably. All this is carefully explained in [23] and [45].

Unfortunately facing general Dirichlet series many of these powerful bridges seem to collapse and new questions arise which make the theory of general Dirichlet quite challenging.

Nevertheless inspired by the ordinary theory we are able to introduce a Fourier analysis setting for the study of general Dirichlet series by restricting ourselves to $\lambda$-Dirichlet series with Dirichlet coefficients that actually are Fourier coefficients defined by functions on compact abelian groups $G$ of a certain type-more precisely, compact abelian groups allowing a continuous homomorphism $\beta: \mathbb{R} \rightarrow G$ with dense range.

2. Bohr's theorem. In order to get started we define the space $\mathcal{D}_{\infty}(\lambda)$ analogously to the space $\mathcal{D}_{\infty}$.

Definition 2.1. Let $\lambda$ be a frequency. Then $\mathcal{D}_{\infty}(\lambda)$ denotes the space of all $\lambda$-Dirichlet series $D$, which converge on $[R e>0]$ and define (a then necessarily holomorphic and) bounded function there.

For instance, if $\lambda=(n)=(0,1,2, \ldots)$, then looking at the transformation $z=e^{-s}$ we easily conclude that $\mathcal{D}_{\infty}((n))$ is simply $H_{\infty}(\mathbb{D})$, the space of all bounded and holomorphic functions on the open unit ball $\mathbb{D}$. And if $\lambda=(\log n)$, then we are in the ordinary case.

Recall that there is a unique coefficient preserving isometry identifying $H_{\infty}(\mathbb{D})$ and $H_{\infty}(\mathbb{T})$. Hence, in both cases, $\lambda=(n)$ and $\lambda=(\log n)$, the space $\mathcal{D}_{\infty}(\lambda)$ can be described in terms of Fourier analysis, that is, it can be considered as a Hardy space, namely $\mathcal{D}_{\infty}((n))=H_{\infty}(\mathbb{T})$ and $\mathcal{D}_{\infty}((\log n))=H_{\infty}\left(\mathbb{T}^{\infty}\right)$. In view of these two examples the fol- 
lowing question arises naturally: Given an arbitrary frequency $\lambda$, is it possible to describe $\mathcal{D}_{\infty}(\lambda)$ in terms of a sort of Hardy space on a compact abelian group?

The first step towards the solution of this problem, is to follow ideas of Bohr and Landau, who asked under which assumptions the abscissa $\sigma_{u}(D)$ can be described in terms of analytic properties of the limit function of $D$. Define

$$
\sigma_{b}(D)=\inf \sigma,
$$

where the infimum is taken over all $\sigma \in \mathbb{R}$ such that $D$ converges and defines a bounded function on $[R e>\sigma]$ (which then is automatically holomorphic). Additionally define, provided $\sigma_{c}(D)<\infty$,

$$
\sigma_{b}^{\operatorname{ext}}(D)=\inf \sigma
$$

the infimum now taken over all $\sigma \in \mathbb{R}$ such that the limit function of $D$ allows a holomorphic and bounded extension to $[R e>\sigma]$. By definition $\sigma_{c}(D) \leq \sigma_{b}(D) \leq \sigma_{u}(D) \leq \sigma_{a}(D)$ and $\sigma_{b}^{\text {ext }}(D) \leq \sigma_{b}(D)$. But in general all these abscissas differ. For instance an example of Bohr from [13] shows that $\sigma_{c}(D)=\sigma_{b}^{\text {ext }}(D)=\sigma_{b}(D)=-\infty$ and $\sigma_{u}(D)=\sigma_{a}(D)=+\infty$ is possible.

A very prominent research project at the beginning of the 20th century was to find conditions for frequencies $\lambda$ under which

$$
\sigma_{b}^{\mathrm{ext}}(D)=\sigma_{u}(D)
$$

holds for all somewhere convergent $\lambda$-Dirichlet series $D$. In this context the space

$$
\mathcal{D}_{\infty}^{\text {ext }}(\lambda)
$$

of all somewhere convergent $\lambda$-Dirichlet series $D=\sum a_{n} e^{-\lambda_{n} s}$, which have a holomorphic and bounded extension to $[R e>0]$, appears naturally. Endow $\mathcal{D}_{\infty}^{\text {ext }}(\lambda)$ with the seminorm $\|D\|_{\infty}:=\sup _{[R e>0]}|f(s)|$, where $f$ is the (unique) extension of $D$. We will later in fact see that this always defines a norm-but that, in general, neither $\mathcal{D}_{\infty}(\lambda)$ nor $\mathcal{D}_{\infty}^{\text {ext }}(\lambda)$ form Banach spaces.

Much of the abstract theory of ordinary Dirichlet series is based on a fundamental theorem of Bohr from [9] which shows that every $D \in \mathcal{D}_{\infty}^{\operatorname{ext}}((\log n))$ converges uniformly on all halfspaces $[R e>\varepsilon]$ for all $\varepsilon>0$, and this then easily implies that $\mathcal{D}_{\infty}^{\text {ext }}((\log n))=$ $\mathcal{D}_{\infty}((\log n))$ (see also [23. Theorem 1.13]). For certain classes of frequencies $\lambda$, Bohr [8] and Landau [42] extend this result to $\lambda$-Dirichlet series.

In [8] Bohr shows that 12 holds if $\lambda$ satisfies the following condition (we call it Bohr's condition $(B C))$ :

$$
\exists l=l(\lambda)>0 \forall \delta>0 \exists C>0 \forall n \in \mathbb{N}: \lambda_{n+1}-\lambda_{n} \geq C e^{-(l+\delta) \lambda_{n}} ;
$$

roughly speaking this condition prevents the $\lambda_{n}$ 's from getting too close too fast. Motivated by Bohr's work we introduce the following definition.

Definition 2.2. We say that a frequency $\lambda$ satisfies Bohr's theorem, whenever every $D \in \mathcal{D}_{\infty}^{\text {ext }}(\lambda)$ converges uniformly on all halfspaces $[R e>\varepsilon], \varepsilon>0$, i.e. $\sigma_{b}^{\text {ext }}(D)=\sigma_{u}(D)$.

Observe that $\lambda=(\log n)$ satisfies $(B C)$ with $l=1$ and so Bohr's theorem holds. In [42] Landau gives a weaker sufficient condition than $(B C)$ (called Landau's condition $(L C)$ ), 
which extends the class of frequencies which satisfy Bohr's theorem:

$$
\forall \delta>0 \exists C>0 \forall n \in \mathbb{N}: \lambda_{n+1}-\lambda_{n} \geq C e^{-e^{\delta \lambda_{n}}} .
$$

We like to mention that in [43, §1] Neder goes a step further and considers $\lambda$ 's satisfying

$$
\exists \delta>0 \exists C>0 \forall n \in \mathbb{N}: \lambda_{n+1}-\lambda_{n} \geq C e^{-e^{\delta \lambda_{n}}} .
$$

Then Neder proves that this condition is not sufficient for Bohr's theorem by constructing, given some $x>0$, a $\lambda$-Dirichlet series $D$ for which $\sigma_{c}(D)=\sigma_{a}(D)=x$ and $\sigma_{b}^{\text {ext }}(D) \leq 0$ holds, which in particular shows that $\mathcal{D}_{\infty}(\lambda) \subsetneq \mathcal{D}_{\infty}^{\text {ext }}(\lambda)$.

Analyzing Bohr's original proof from [8], Bohr's theorem in the ordinary case is improved in [3] by a quantitative version (see also [23, Theorem 1.13] or [45, Theorem $6.2 .2])$.

THEOREM 2.3. There is a constant $C>0$ such that for every $D \in \mathcal{D}_{\infty}^{\operatorname{ext}}((\log n))$ and all $N \geq 2$

$$
\sup _{[\operatorname{Re}>0]}\left|\sum_{n=1}^{N} a_{n} n^{-s}\right| \leq C \log (N)\|D\|_{\infty} .
$$

Note that inequality (15) together with 11 implies 12 for $\lambda=(\log n)$.

Like in the work of Bohr, Landau only proves the qualitative version of the fact that each frequency $\lambda$ under his condition $(L C)$ satisfies Bohr's theorem. To establish quantitative versions in the sense of 15 means to control the norm of the partial sum operator

$$
S_{N}: \mathcal{D}_{\infty}^{\text {ext }}(\lambda) \rightarrow \mathcal{D}_{\infty}(\lambda), \quad D \mapsto \sum_{n=1}^{N} a_{n}(D) e^{-\lambda_{n} s} .
$$

Using the summation method of typical means of order $k>0$ invented by M. Riesz (see Proposition 2.6, the following estimate of $\left\|S_{N}\right\|$, which holds under no further conditions on $\lambda$, is the main result of [48, Theorem 3.2].

TheOrem 2.4. Let $D=\sum a_{n} e^{-\lambda_{n} s} \in \mathcal{D}_{\infty}^{\text {ext }}(\lambda)$. Then for all $0<k \leq 1$ and $N \in \mathbb{N}$ :

$$
\sup _{[\operatorname{Re}>0]}\left|\sum_{n=1}^{N} a_{n} e^{-\lambda_{n} s}\right| \leq C \frac{\Gamma(k+1)}{k}\left(\frac{\lambda_{N+1}}{\lambda_{N+1}-\lambda_{N}}\right)^{k}\|D\|_{\infty},
$$

where $C>0$ is a universal constant and $\Gamma$ denotes the Gamma function.

As a consequence, assuming Bohr's condition $(B C)$ for $\lambda$, the choice $k_{N}:=\frac{1}{\lambda_{N}}, N \geq 2$ (note that $\lambda_{1}=0$ is possible), leads to

$$
\left\|S_{N}: \mathcal{D}_{\infty}^{\mathrm{ext}}(\lambda) \rightarrow \mathcal{D}_{\infty}(\lambda)\right\| \leq C_{1}(\lambda) \lambda_{N}
$$

which reproves 15 for $\lambda=(\log n)$. If we assume Landau's condition $(L C)$, the estimate from Theorem 2.4 with $k_{N}:=e^{-\delta \lambda_{N}}, \delta>0$, gives

$$
\left\|S_{N}: \mathcal{D}_{\infty}^{\text {ext }}(\lambda) \rightarrow \mathcal{D}_{\infty}(\lambda)\right\| \leq C_{2}(\lambda, \delta) e^{\delta \lambda_{N}}
$$

the quantitative version of Bohr's theorem under $(L C)$.

For particular frequencies $\lambda$ the bounds from (16) and 17) may be bad, which is not too surprising since these results in fact hold for all frequencies satisfying the conditions 
of Bohr or Landau. For instance, consider the case $\lambda=(n)$. Then the projection

$$
S_{N}: H_{\infty}(\mathbb{T}) \rightarrow H_{\infty}(\mathbb{T}), \quad S_{N}(f)=\sum_{n=0}^{N} \widehat{f}(n) z^{n}
$$

is nothing else than the convolution operator which assigns to every $f$ its convolution with the Dirichlet kernel $D_{N}$, and this immediately gives $\left\|S_{N}\right\|=\left\|D_{N}\right\|_{1} \sim \log (N)$. To our best knowledge the optimal upper and lower bounds for the norm of $S_{N}$ in the ordinary case $\lambda=(\log n)$ are still unknown.

Problem 2.5. Determine optimal bounds for $\left\|S_{N}: \mathcal{D}_{\infty}((\log n)) \rightarrow \mathcal{D}_{\infty}((\log n))\right\|$.

The proof of Theorem 2.4 relies on the following independently interesting result from [48, Proposition 3.4] which was inspired by the work of Hardy and M. Riesz from [36].

Proposition 2.6. Let $D=\sum a_{n} e^{-\lambda_{n} s} \in D_{\infty}^{\text {ext }}(\lambda)$ with extension $f$. Then for all $k>0$ the Dirichlet polynomials

$$
R_{x}^{k}(D)=\sum_{\lambda_{n}<x} a_{n}\left(1-\frac{\lambda_{n}}{x}\right)^{k} e^{-\lambda_{n} s}
$$

converge uniformly to $f$ on $[$ Re $>\varepsilon]$ for all $\varepsilon>0$ as $x \rightarrow \infty$. Moreover, for some universal constant $C>0$

$$
\sup _{x \geq 0}\left\|R_{x}^{k}(D)\right\|_{\infty} \leq C \frac{\Gamma(k+1)}{k}\|D\|_{\infty} .
$$

In the language of 36 this proposition states that, given any order $k>0$, on every halfplane $[R e>\varepsilon]$ the limit function of a Dirichlet series $D \in \mathcal{D}_{\infty}^{\text {ext }}(\lambda)$ is the uniform limit of its first typical means of order $k$.

Moreover, Proposition 2.6 gives a direct link to the theory of almost periodic functions on $\mathbb{R}$, and proves that $\mathcal{D}_{\infty}^{\text {ext }}(\lambda)$ in fact is a normed space (see Corollary 2.8). Note that a priori, $\|\cdot\|_{\infty}$ is only a seminorm, or equivalently, it is not clear whether $\mathcal{D}_{\infty}^{\text {ext }}(\lambda)$ can be considered as a subspace of $H_{\infty}[R e>0]$, the Banach space of all holomorphic and bounded functions on $[R e>0]$. Here it is important to distinguish Dirichlet series from their limit function, and to prove that $\|\cdot\|_{\infty}$ in fact is a norm on $\mathcal{D}_{\infty}^{\text {ext }}(\lambda)$ requires to check that all Dirichlet coefficients of $D$ vanish provided $\|f\|_{\infty}=0$.

Recall that by definition a continuous function $f: \mathbb{R} \rightarrow \mathbb{C}$ is called (uniformly) almost periodic, whenever for every $\varepsilon>0$ there is a number $l>0$ such that for all intervals $I \subset \mathbb{R}$ with $|I|=l$ there is a translation number $\tau \in I$ such that $\sup _{x \in \mathbb{R}}|f(x+\tau)-f(x)| \leq \varepsilon$ (see [6] for more information). Then by a result of Bohr a bounded and continuous function $f$ is almost periodic if and only if it is the uniform limit of trigonometric polynomials on $\mathbb{R}$, which are of the form $p(t)=\sum_{n=1}^{N} a_{n} e^{-i t x_{n}}$ for some $x_{1}, \ldots, x_{N} \in \mathbb{R}$ (see e.g. [45, Theorem 1.5.5, p. 25]). In particular, the Dirichlet polynomials $R_{x}^{k}(D)$ from Proposition 2.6 considered as functions on vertical lines $[R e=\sigma]$ are almost periodic.

COROLlary 2.7. Let $D \in \mathcal{D}_{\infty}^{\text {ext }}(\lambda)$ with an arbitrary frequency $\lambda$ and extension $f$. Then for all $\sigma>0$ the function $f_{\sigma}(t):=f(\sigma+i t): \mathbb{R} \rightarrow \mathbb{C}$ is almost periodic and

$$
a_{n}(D)=\lim _{T \rightarrow \infty} \frac{1}{2 T} \int_{-T}^{T} f(\sigma+i t) e^{(\sigma+i t) \lambda_{n}} .
$$

In particular, $\sup _{n \in \mathbb{N}}\left|a_{n}\right| \leq\|D\|_{\infty}$. 
This result is taken from [48, Corollary 3.8], and the next corollary is then an immediate consequence.

COROLlary 2.8. $\mathcal{D}_{\infty}^{\text {ext }}(\lambda)$, and consequently also its subspace $\mathcal{D}_{\infty}(\lambda)$, are normed spaces for any frequency $\lambda$.

Another particular consequence of Corollary 2.7 is as follows. Clearly, we may deduce from this corollary that $\left\|S_{N}: \mathcal{D}_{\infty}^{\text {ext }}(\lambda) \rightarrow \mathcal{D}_{\infty}(\lambda)\right\| \leq N$ for all $N$, so equality (12) follows from (11), i.e. $\lambda$ satisfies Bohr's theorem, whenever

$$
L(\lambda):=\limsup _{N \rightarrow \infty} \frac{\log (N)}{\lambda_{N}}=0 .
$$

We like to mention that the number $L(\lambda)$ has the following geometric meaning in terms of abscissas. In [10, $§ 3$, Hilfssatz 2 and 3] Bohr proves that

$$
L(\lambda)=\sigma_{c}\left(\sum e^{-\lambda_{n} s}\right)=\sigma_{a}\left(\sum e^{-\lambda_{n} s}\right)=\sup _{D \in \mathcal{D}(\lambda)} \sigma_{a}(D)-\sigma_{c}(D) .
$$

For instance $L((n))=0$, and we obtain as a consequence that for power series we up to $\varepsilon$ cannot distinguish between uniform convergence and boundedness of the limit function.

Besides $\lambda$ 's with $(B C)$ or $(L C)$, there is another class of frequencies $\lambda$ for which Bohr's theorem holds. In [1] Bohr proves that $\mathbb{Q}$-linearly independent frequencies $\lambda$ satisfy

$$
\sigma_{b}^{\operatorname{ext}}(D)=\sigma_{a}(D)
$$

for all somewhere convergent $\lambda$-Dirichlet series $D$. The use of Kronecker's theorem, which states that the set $\left\{\left(e^{-\lambda_{n} i t}\right) \mid t \in \mathbb{R}\right\}$ is dense in $\mathbb{T}^{\infty}$ whenever the real sequence $\left(\lambda_{n}\right)$ is $\mathbb{Q}$-linearly independent, combined with Proposition 2.6 lead to an alternative proof of this fact.

THEOREM 2.9. Let $D=\sum a_{n} e^{-\lambda_{n} s} \in \mathcal{D}_{\infty}^{\text {ext }}(\lambda)$ with a $\mathbb{Q}$-linearly independent frequency $\lambda$. Then $\left(a_{n}\right) \in \ell_{1}$ and $\left\|\left(a_{n}\right)\right\|_{1}=\|D\|_{\infty}$. Moreover isometrically,

$$
\mathcal{D}_{\infty}^{\text {ext }}(\lambda)=\mathcal{D}_{\infty}(\lambda)=\ell_{1}, \quad \sum a_{n} e^{-\lambda_{n} s} \mapsto\left(a_{n}\right)
$$

and $\lambda$ satisfies Bohr's theorem. In particular,

$$
\sup _{N \in \mathbb{N}} \sup _{[\operatorname{Re}>0]}\left|\sum_{n=1}^{N} a_{n} e^{-\lambda_{n} s}\right|=\|D\|_{\infty} .
$$

The following theorem summarizes some of the preceding results on frequencies $\lambda$ satisfying Bohr's theorem.

THEOREM 2.10. A frequency $\lambda$ satisfies Bohr's theorem, whenever one of following conditions holds:

- $L(\lambda)=0$,

- $\lambda$ is $\mathbb{Q}$-linearly independent,

- $\lambda$ satisfies $(L C)$ (or the stronger condition $(B C)$ ).

Moreover, in each of these cases we have $\mathcal{D}_{\infty}^{\mathrm{ext}}(\lambda)=\mathcal{D}_{\infty}(\lambda)$, but none of these conditions is necessary for Bohr's theorem.

Unfortunately $\mathcal{D}_{\infty}(\lambda)$ may fail to be a Banach space. The following result from [48, Theorem 5.2] is inspired by a construction of Neder given in [43, $\S 1$ ]. 
TheOREM 2.11. Let $\lambda=\left(\lambda_{n}\right)$ be a frequency. Then there is a strictly increasing sequence $\left(s_{n}\right)$ of natural numbers such that $\mathcal{D}_{\infty}(\eta)$, where $\eta$ is the frequency obtained by ordering the set

$$
\left\{\lambda_{n}+\frac{j}{s_{n}}\left(\lambda_{n+1}-\lambda_{n}\right) \mid n \in \mathbb{N}, j=0, \ldots, s_{n}-1\right\}
$$

increasingly, is not complete and Bohr's theorem fails for $\eta$.

Following the proof of [48, Theorem 5.2] the particular case $\lambda=(n)$ recovers Neder's construction from [43, §1] and gives the existence of a frequency $\eta$ for which the inclusion $\mathcal{D}_{\infty}(\eta) \subset \mathcal{D}_{\infty}^{\text {ext }}(\eta)$ is strict. Moreover, a modification of Neder's proof gives a frequency $\eta$ for which $\mathcal{D}_{\infty}^{\text {ext }}(\eta)$ does not form a Banach space. The good news is that completeness of $\mathcal{D}_{\infty}(\lambda)$ and $\mathcal{D}_{\infty}^{\text {ext }}(\lambda)$ is guaranteed by sufficient conditions on $\lambda$ which in concrete cases have a good chance to be checked.

The following important result is proven in [48, Theorem 5.1] and [28, Theorem 5.1], which in particular shows that $\mathcal{D}_{\infty}(\lambda)$ is complete under all three concrete conditions mentioned in Theorem 2.10 (for the case of $(B C)$ see also [20]).

THEOREM 2.12. Let $\lambda$ be an arbitrary frequency.

(a) $\mathcal{D}_{\infty}^{\text {ext }}(\lambda)$ is complete, if $L(\lambda)<\infty$.

(b) $\mathcal{D}_{\infty}(\lambda)$ is a Banach space if and only if $\lambda$ satisfies Bohr's theorem. Moreover, in this case $\mathcal{D}_{\infty}^{\text {ext }}(\lambda)=\mathcal{D}_{\infty}(\lambda)$.

This result shows in particular that $\mathcal{D}_{\infty}^{\text {ext }}(\lambda)$ is a Banach space, whenever $\mathcal{D}_{\infty}(\lambda)$ is complete.

Problem 2.13. Assume that $\mathcal{D}_{\infty}^{\text {ext }}(\lambda)$ is complete. Does this necessarily imply that $\mathcal{D}_{\infty}(\lambda)$ is complete or equivalently Bohr's theorem holds for $\lambda$ ?

According to Theorem 2.12, finding some $\lambda$ such that $L(\lambda)<\infty$ and Bohr's theorem fails, would show that completeness of $\mathcal{D}_{\infty}^{\text {ext }}(\lambda)$ in general does not imply completeness of $\mathcal{D}_{\infty}(\lambda)$. In this context more interesting questions appear naturally - even for very concrete frequencies.

Problem 2.14. Let $\lambda=(\log (\log n))$. Does $\lambda$ satisfy Bohr's theorem? Equivalently: Is $\mathcal{D}_{\infty}(\lambda)$ complete?

Recall Theorem 2.10 which states conditions on frequencies under which Bohr's theorem holds and so consequently $\sigma_{b}^{\operatorname{ext}}(D) \geq \sigma_{c}(D)$ for all $\lambda$-Dirichlet series $D$.

Problem 2.15. Find a reasonable condition on the frequency $\lambda$ which is weaker than $(L C)$, but is sufficient for $\sigma_{b}^{\mathrm{ext}}(D) \geq \sigma_{c}(D)$ for all $\lambda$-Dirichlet series $D$.

We finish summarizing a few relations of the three conditions $L(\lambda)<\infty,(B C)$ and $(L C)$ (see [48, Remark 4.1]).

REMARK 2.16.

- $(B C)$ implies $L(\lambda)<\infty$ and $(L C)$.

- $(L C)$ plus $L(\lambda)<\infty$ does not necessarily imply $(B C)$.

- $L(\lambda)<\infty$ does not necessarily imply $(L C)$ and so neither $(B C)$.

- $(L C)$ does not necessarily imply $L(\lambda)<\infty$ and so neither $(B C)$. 
3. Hardy spaces of general Dirichlet series. In the following section we sketch our $\mathcal{H}_{p}$-theory for general Dirichlet series. As already mentioned in the introduction we restrict ourselves to general Dirichlet series with Dirichlet coefficients which actually are Fourier coefficients of functions on certain compact abelian groups. This has several advantages. One is that the class of all general Dirichlet series simply is too large to obtain a good understanding. Assuming that the Dirichlet coefficients are Fourier coefficients gives more structure and allows using tools from harmonic analysis like the HausdorffYoung inequality or Plancherel's theorem (among others). A further advantage of our setting is that Bayart's $\mathcal{H}_{p}$-theory of ordinary Dirichlet series embeds in a natural way. Whereas the $\mathcal{H}_{p}$-theory of ordinary Dirichlet series is basically Fourier analysis on the infinite dimensional torus $\mathbb{T}^{\infty}$, this group fails to be the right model for general Dirichlet series.

In fact, for any frequency $\lambda$ the Bohr compactification $\overline{\mathbb{R}}$ of $\mathbb{R}$ or products of $\widehat{\mathbb{Q}_{d}}$ (the dual group of the rationals endowed with the discrete topology) turn out to be suitable substitutes. But if we fix some $\lambda$, then on one hand there might be a natural group adapted to it (as $\mathbb{T}^{\infty}$ in the ordinary case), although on the other hand an important feature of our approach is that the Hardy spaces of $\lambda$-Dirichlet series we intend to define, at least as Banach spaces are independent of the chosen suitable group for $\lambda$.

We consider the subclass of all compact abelian groups $G$ which allow a continuous homomorphism $\beta: \mathbb{R} \rightarrow G$ with dense range, and call such pairs $(G, \beta)$ Dirichlet groups. Hence, given such a pair, the characters $e^{-i x} \in \widehat{\beta}(\widehat{G}) \subset \widehat{\mathbb{R}}$ are precisely those for which there is a unique character $h_{x} \in \widehat{G}$ with $e^{-i x}=h_{x} \circ \beta$; recall here that $\mathbb{R}=\widehat{\mathbb{R}}, x \mapsto e^{-i x}$, is a group isomorphism. In particular, we have

$$
\widehat{G}=\left\{h_{x} \mid x \in \widehat{\beta}(\widehat{G})\right\} .
$$

Then the following notion (first given in [26]) turns out to be fundamental for our purposes.

Definition 3.1. Let $\lambda$ be a frequency and $(G, \beta)$ a Dirichlet group. Then $(G, \beta)$ is called a $\lambda$-Dirichlet group whenever $\lambda \subset \widehat{\beta}(\widehat{G})$, which means that the following diagram commutes for every $n \in \mathbb{N}$ :

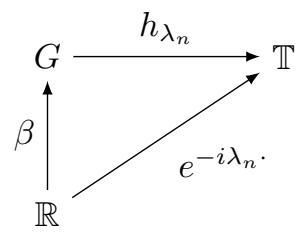

Let us give examples. Denoting by $\mathfrak{p}:=\left(p_{n}\right)$ the sequence of prime numbers, the compact group $\mathbb{T}^{\infty}$ together with the mapping

$$
\beta_{\mathbb{T}^{\infty}}: \mathbb{R} \rightarrow \mathbb{T}^{\infty}, \quad t \mapsto \mathfrak{p}^{-i t}
$$

forms a $(\log n)$-Dirichlet group (see again Kronecker's theorem). This example keeps us in track to recover results on ordinary Dirichlet series. The 'mother' of all possible examples is as follows: Given a subgroup $U$ of $\mathbb{R}$, the topological group $\widehat{(U, d)}$, where $d$ denotes the 
discrete topology, together with the mapping

$$
\beta_{\widehat{(U, d)}}: \mathbb{R} \rightarrow \widehat{(U, d)}, \quad t \mapsto\left[u \mapsto e^{-i t u}\right]
$$

forms a Dirichlet group. So in particular, for $U=\mathbb{Z}$ and identifying $\mathbb{T}=\widehat{\mathbb{Z}}$, we obtain the $(n)$-Dirichlet group $\left(\mathbb{T}, \beta_{\mathbb{T}}\right)$, where

$$
\beta_{\mathbb{T}}: \mathbb{R} \rightarrow \mathbb{T}, \quad t \mapsto e^{-i t} .
$$

The compact abelian group $\overline{\mathbb{R}}:=\widehat{(\mathbb{R}, d)}$ is the so-called Bohr compactification of $\mathbb{R}$ which forms a $\lambda$-Dirichlet group for all frequencies $\lambda$ with the embedding

$$
\beta_{\overline{\mathbb{R}}}:(\mathbb{R},|\cdot|) \hookrightarrow \overline{\mathbb{R}}, \quad x \mapsto\left[t \mapsto e^{-i x t}\right] .
$$

So the pair $\left(\overline{\mathbb{R}}, \beta_{\overline{\mathbb{R}}}\right)$ is a sort of 'universal' Dirichlet group. But also the countable product of $\widehat{\mathbb{Q}_{d}}:=\widehat{(\mathbb{Q}, d)}$ in a slightly different sense forms a Dirichlet group for all frequencies. To explain this, let $B=\left(b_{1}, b_{2}, \ldots\right)$ be a $\mathbb{Q}$-linearly independent sequence of real numbers of length $N \in \mathbb{N} \cup\{\infty\}$. Then

$$
T_{B}: \bigoplus_{n=1}^{N} \mathbb{Q} \hookrightarrow \mathbb{R}, \quad \alpha \mapsto \sum \alpha_{j} b_{j}
$$

is an injective homomorphism, and hence its dual map

$$
\widehat{T_{B}}: \mathbb{R} \rightarrow \widehat{\bigoplus_{n=1}^{N} \mathbb{Q}_{d}}, \quad t \mapsto\left[\left(q_{j}\right)_{j} \mapsto e^{-i t \sum q_{j} b_{j}}\right]
$$

has dense range. Since $\prod_{n=1}^{N} \widehat{\mathbb{Q}}_{d}=\bigoplus_{n=1}^{N} \mathbb{Q}_{d}$, the pair $\left(\prod_{n=1}^{N} \widehat{\mathbb{Q}_{d}}, \widehat{T_{B}}\right)$ is a Dirichlet group, which is 'universal' in the following sense: For every frequency $\lambda$ there is a suitable $B$ such that $\left(\prod_{n=1}^{N} \widehat{\mathbb{Q}_{d}}, \widehat{T_{B}}\right)$ is a $\lambda$-Dirichlet group. To see this, let us recall that for every $\lambda$ there is a $\mathbb{Q}$-linearly independent sequence $B=\left(b_{k}\right)$ of real numbers of length $N \in \mathbb{N} \cup\{\infty\}$, called basis for $\lambda$ (which can always be chosen as a subsequence of $\lambda$ ), such that $\lambda_{n}=\sum r_{k}^{n} b_{k}$ for some (unique) finite rational sequence $\left(r_{k}^{n}\right)$. In this case, $R=\left(r_{k}^{n}\right)_{n, k}$ is said to be a Bohr matrix of $\lambda$ with respect to the basis $B$ and we write $\lambda=(R, B)$. Hence $\left(\prod_{n=1}^{N} \widehat{\mathbb{Q}_{d}}, \widehat{T_{B}}\right)$ is a $\lambda$-Dirichlet group for every $\lambda$ with decomposition $\lambda=(R, B)$.

Given a $\lambda$-Dirichlet group $(G, \beta)$ and $1 \leq p \leq \infty$, we define the Banach space

$$
H_{p}^{\lambda}(G):=\left\{f \in L_{p}(G) \mid \widehat{f}: \widehat{G} \rightarrow \mathbb{C} \text { is supported by all } h_{\lambda_{n}}, n \in \mathbb{N}\right\},
$$

and use it to define the following natural scale of Hardy spaces of general Dirichlet series.

Definition 3.2. The Hardy space $\mathcal{H}_{p}(\lambda)$ consists of all $\lambda$-Dirichlet series $\sum a_{n} e^{-\lambda_{n} s}$ for which there is some (unique) $f \in H_{p}^{\lambda}(G)$ such that $a_{n}=\widehat{f}\left(h_{\lambda_{n}}\right)$ for all $n$.

Together with the norm $\|D\|_{p}:=\|f\|_{p}$ the space $\mathcal{H}_{p}(\lambda)$ clearly forms a Banach space, and then by definition the Bohr map

$$
\mathcal{B}: H_{p}^{\lambda}(G) \hookrightarrow \mathcal{D}(\lambda), \quad f \sim \sum_{\gamma \in \widehat{G}} \widehat{f}(\gamma) \gamma \mapsto \sum_{n \in \mathbb{N}} \widehat{f}\left(h_{\lambda_{n}}\right) e^{-\lambda_{n} s}
$$

gives an isometric onto isomorphism,

$$
\mathcal{H}_{p}(\lambda)=H_{p}^{\lambda}(G) .
$$


The following fact, proved in [26, Theorem 3.19], is fundamental.

TheOREm 3.3. $\mathcal{H}_{p}(\lambda)$ is independent of the chosen $\lambda$-Dirichlet group $(G, \beta)$.

In particular, in the ordinary case the Banach space $\mathcal{H}_{p}((\log n))$ actually coincides with Bayart's Banach space $\mathcal{H}_{p}$ from [4] (see also [23, Chapter 11]). Moreover, recall that in this case the groups $\mathbb{T}^{\infty}$ and $\overline{\mathbb{R}}$ are suitable $(\log n)$-Dirichlet groups, which immediately leads to the following consequence extending $(3)$.

Corollary 3.4. For all $1 \leq p \leq \infty$ we have

$$
H_{p}^{(\log n)}(\overline{\mathbb{R}})=\mathcal{H}_{p}((\log n))=H_{p}\left(\mathbb{T}^{\infty}\right), \quad\|f\|_{p}=\|D\|_{p}=\|g\|_{p}
$$

where $\widehat{f}\left(h_{\log n}\right)=a_{n}(D)=\widehat{g}(\alpha)$, whenever $n=\mathfrak{p}^{\alpha}$.

Let us mention that there also is an internal description of $\mathcal{H}_{p}(\lambda)$ through $\lambda$-Dirichlet polynomials without considering $\lambda$-Dirichlet groups. We denote by $\operatorname{Pol}(\lambda)$ the space of all $\lambda$-Dirichlet polynomials $D(s)=\sum_{n=1}^{N} a_{n} e^{-\lambda_{n} s}$. For such polynomials we define

$$
\|D\|_{p}:=\left(\lim _{T \rightarrow \infty} \frac{1}{2 T} \int_{-T}^{T}\left|\sum_{n=1}^{N} a_{n} e^{-\lambda_{n} i t}\right|^{p} d t\right)^{1 / p} .
$$

Then this limit exists and gives a norm on $\operatorname{Pol}(\lambda)$ (see e.g. [45, Theorem 1.5.6, p. 26] or for the ordinary case [23, Theorem 11.9]). The following observation is taken from [26, Theorem 3.25].

THEOREM 3.5. Let $1 \leq p<\infty$ and $\lambda$ a frequency. Then the space $\mathcal{H}_{p}(\lambda)$ is the completion of $\left(\operatorname{Pol}(\lambda),\|\cdot\|_{p}\right)$.

3.1. Frequencies of integer type. Recall that given a frequency $\lambda$ there is a basis $B$ for $\lambda$ and a Bohr matrix $R$ such that $\lambda=(R, B)$. Let us look at the ordinary case $\lambda=(\log n)$. Then $\log n=\sum \alpha_{j} \log p_{j}$ whenever $n=\mathfrak{p}^{\alpha}$, and hence a basis is given by $B=\left(\log p_{j}\right)$ and every multi-index $\alpha>0$ appears as a row in the corresponding Bohr matrix $R$. Moreover recall that $\mathbb{T}^{\infty}$ is a $(\log n)$-Dirichlet group with $\beta_{\mathbb{T}^{\infty}}: \mathbb{R} \rightarrow \mathbb{T}^{\infty}$, $\beta_{\mathbb{T}^{\infty}}(t)=\mathfrak{p}^{-i t}$.

More generally, we call a frequency $\lambda$ of integer (natural) type, if there is a basis $B=\left(b_{n}\right)$ such that the Bohr matrix $R$ associated to $B$ and $\lambda$ only has integer (natural) entries. In this case $\mathbb{T}^{\infty}$ is a $\lambda$-Dirichlet group with $\beta(t):=e^{-i t B}:=\left(e^{-i t b_{1}}, e^{-i t b_{2}}, \ldots\right)$, $t \in \mathbb{R}$, and the rows of the corresponding Bohr matrix are multi-indices $\alpha$ (with integer entries). Actually one can show that a frequency $\lambda$ is of integer type if and only if there is a homomorphism $\beta: \mathbb{R} \rightarrow \mathbb{T}^{\infty}$ such that $\left(\mathbb{T}^{\infty}, \beta\right)$ is a $\lambda$-Dirichlet group (see [26. Remark 3.32]).

To see an example, consider the set $M:=\{n+\sqrt{2} m \mid n, m \in \mathbb{Z}\}$ which is dense in $\mathbb{R}$. Then any frequency $\lambda \subset M$ is of integer type with basis $B=(1, \sqrt{2})$. See $[26$, Example 3.37] for a frequency which is not of integer type.

Let $B$ be a basis of length $N \in \mathbb{N} \cup\{\infty\}$ for some frequency $\lambda$ of integer type with Bohr matrix $R$, and let us write $\alpha \in R$ if the multi-index $\alpha$ appears as a row in $R$. Then we define $H_{p}^{R}\left(\mathbb{T}^{N}\right)$ to be the Banach space of all $g \in L_{p}\left(\mathbb{T}^{N}\right)$ for which $\widehat{g}(\alpha) \neq 0$ implies $\alpha \in R$. Using Theorem 3.3, we see that for such frequencies $\lambda$ the $\mathcal{H}_{p}(\lambda)$ 's in the following sense may be identified with $H_{p}^{R}\left(\mathbb{T}^{N}\right)$ (see [26, Theorem 3.31]). 
Theorem 3.6. Let $1 \leq p \leq \infty$ and $\lambda=(R, B)$ a frequency of integer type with basis of length $N \in \mathbb{N} \cup\{\infty\}$. Then there is a unique onto isometry

$$
\psi: \mathcal{H}_{p}(\lambda) \rightarrow H_{p}^{R}\left(\mathbb{T}^{N}\right), \quad D \mapsto g
$$

such that $\widehat{g}(\alpha)=a_{n}(D)$ for all multi-indices $\alpha \in R$ and $\lambda_{n}=\sum \alpha_{j} b_{j}$.

An immediate consequence is that the ordinary $\mathcal{H}_{p}$ 's in the following sense are the largest spaces for $\lambda$ 's of natural type.

Corollary 3.7. Let $1 \leq p \leq \infty$ and $\lambda=(R, B)$ a frequency of natural type. Then there is a unique into isometry

$$
\psi: \mathcal{H}_{p}(\lambda) \hookrightarrow \mathcal{H}_{p}, \quad \sum a_{n} e^{-\lambda_{n} s} \rightarrow \sum b_{n} n^{-s}
$$

such that $a_{n}=b_{\mathfrak{p}^{\alpha}}$ for all $\alpha \in R$, where $\lambda_{n}=\sum \alpha_{j} b_{j}$.

4. Some structure theory. We summarize further properties of $\mathcal{H}_{p}(\lambda)$ which extend important keystones from Bayart's theory of ordinary Dirichlet series to our new theory of general Dirichlet series.

4.1. Coincidence. Note first that there are now two ' $H_{\infty}$-spaces of $\lambda$-Dirichlet series' around, namely $\mathcal{D}_{\infty}(\lambda)$ and $\mathcal{H}_{\infty}(\lambda)$. Recall that by Theorem 2.11 there are frequencies $\lambda$ such that $\mathcal{D}_{\infty}(\lambda)$ is not complete, hence in these cases $\mathcal{D}_{\infty}(\lambda) \neq \mathcal{H}_{\infty}(\lambda)$. We collect the results given in [26, $\S 4.12$, Theorem 4.10], [28, Theorem 5.1] and [29, Corollary 2.17], which together yield a far reaching extension of the Bohr-Hedenmalm-Lindqvist-Seip theorem from (8).

Theorem 4.1. For every frequency $\lambda$ with $\lambda$-Dirichlet group $(G, \beta)$ there is an into isometry

$$
\mathcal{D}_{\infty}^{\text {ext }}(\lambda) \hookrightarrow H_{\infty}^{\lambda}(G), \quad D \mapsto f
$$

such that $a_{n}(D)=\widehat{f}\left(h_{\lambda_{n}}\right)$ for all $n$. Moreover, the equality $\mathcal{D}_{\infty}^{\text {ext }}(\lambda)=\mathcal{H}_{\infty}(\lambda)$ holds isometrically if and only $\mathcal{D}_{\infty}^{\text {ext }}(\lambda)$ is complete. The same result is true, if we replace $\mathcal{D}_{\infty}^{\text {ext }}(\lambda)$ by $\mathcal{D}_{\infty}(\lambda)$.

Together with Theorem 2.12 we obtain the following immediate consequence.

COROLlary 4.2. Let $\lambda$ be an arbitrary frequency. Then the following are equivalent:

(a) Bohr's theorem holds for $\lambda$,

(b) $\mathcal{D}_{\infty}(\lambda)$ is complete,

(c) $\mathcal{D}_{\infty}(\lambda)=\mathcal{H}_{\infty}(\lambda)$ holds isometrically.

Moreover, in this case $\mathcal{D}_{\infty}^{\text {ext }}(\lambda)=\mathcal{H}_{\infty}(\lambda)$.

An interesting by-product of Theorem 4.1 is an immediate consequence of the definition of $\mathcal{H}_{2}(\lambda)$ and Parseval's equality, and reads as follows.

Corollary 4.3. For each $D \in \mathcal{D}_{\infty}^{\text {ext }}(\lambda)$ we have $\left(a_{n}(D)\right) \in \ell_{2}$ with $\left\|\left(a_{n}(D)\right)\right\|_{2} \leq\|D\|_{\infty}$, i.e. the embedding

$$
\mathcal{D}_{\infty}^{\text {ext }}(\lambda) \subset \mathcal{H}_{2}(\lambda)
$$

is a well-defined contraction. 
4.2. Schauder bases. Given a frequency $\lambda$ and $1<p<\infty$, the following question is fundamental: Do the $e^{-\lambda_{n} s}$ form a Schauder basis for $\mathcal{H}_{p}(\lambda)$ ? In the ordinary case $\lambda=(\log n)$ the answer is affirmative as discovered in [1], and we will see that the same result is true for arbitrary frequencies $\lambda$.

In 22 we note that, given a Dirichlet group $(G, \beta)$, we have

$$
\widehat{G}=\left\{h_{x} \mid x \in \widehat{\beta}(\widehat{G})\right\} .
$$

This in particular shows that the dual group $\widehat{G}$ inherits the order of $\mathbb{R}$, and hence we deduce from [47, Theorem 8.7.2, p. 217], that the 'Riesz projection'

$$
\Phi\left(\sum a_{k} h_{x_{k}}\right):=\sum_{x_{k} \geq 0} a_{k} h_{x_{k}}
$$

is bounded on the subspace $\operatorname{Pol}(G)$ of all polynomials in $L_{p}(G), 1<p<\infty$. Then standard arguments show the following important theorem from [26, Theorem 4.16].

THEOREM 4.4. Let $1<p<\infty$ and $\lambda$ be a frequency. Then the monomials $\left(e^{-\lambda_{n} s}\right)$ form a Schauder basis for $\mathcal{H}_{p}(\lambda)$.

An equivalent formulation of Theorem 4.4 is that for $1<p<\infty$ all projections

$$
S_{N}^{p}: \mathcal{H}_{p}(\lambda) \rightarrow \mathcal{H}_{p}(\lambda), \quad \sum a_{n} e^{-\lambda_{n} s} \mapsto \sum_{n=1}^{N} a_{n} e^{-\lambda_{n} s}
$$

are uniformly bounded with respect to $N$. But for the border cases $p=1$ and $p=\infty$ this in general is false (e.g. for the frequencies $\lambda=(\log n)$ or $\lambda=(n))$. Upper bounds for the growth of the partial sum operators in $\mathcal{D}_{\infty}(\lambda)$ (which is $\mathcal{H}_{\infty}(\lambda)$, if $\lambda$ has e.g. $(L C)$ ) were given in (16) and (17). The following result handles the case $p=1$, and its proof in a sense reduces to the case $p=\infty$.

Proposition 4.5. Let $\lambda=\left(\lambda_{n}\right)$ be a frequency. Assuming $(B C)$ for $\lambda$ there is a constant $C_{1}=C_{1}(\lambda)$ such that for all $N \geq 2$

$$
\left\|S_{N}^{1}: \mathcal{H}_{1}(\lambda) \rightarrow \mathcal{H}_{1}(\lambda)\right\| \leq C_{1} \lambda_{N}
$$

and, assuming $(L C)$ for every $\delta$ there is a constant $C_{2}=C_{2}(\delta, \lambda)$ such that for all $N$

$$
\left\|S_{N}^{1}: \mathcal{H}_{1}(\lambda) \rightarrow \mathcal{H}_{1}(\lambda)\right\| \leq C_{2} e^{\delta \lambda_{N}} .
$$

A proof is given in [26, Proposition 4.17 and Lemma 4.9]. Note that there the assumption $L(\lambda)<\infty$ appears to be superfluous, since $\mathcal{D}_{\infty}(\lambda)$ already is complete, whenever $\lambda$ satisfies $(L C)$ (see [28, Theorem 5.1] or Theorem 2.12). For the ordinary case see e.g. [23, $\S 12.5]$.

4.3. Brothers Riesz theorem. Recall the classical brothers Riesz theorem, which states that the Hardy space $\mathcal{H}_{1}((n))=H_{1}(\mathbb{T})$ coincides with the space $M(\mathbb{T})$ of all bounded, regular and analytic Borel measures on $\mathbb{T}$. The corresponding result in the ordinary case reads $\mathcal{H}_{1}((\log n))=H_{1}\left(\mathbb{T}^{\infty}\right)=M\left(\mathbb{T}^{\infty}\right)$ (due to Helson and Lowdenslager in [41]). See also [23, Theorem 13.5] for a proof within the setting of ordinary Dirichlet series. The brothers Riesz theorem extends to the case of general Dirichlet series. Denote by $M_{\lambda}(G)$ the space of all bounded and regular Borel measures $\mu$ on $G$ such that $\widehat{\mu}\left(h_{x}\right) \neq 0$ implies $x=\lambda_{n}$ for some $n$. 
Theorem 4.6. Let $\lambda$ be any frequency and $(G, \beta)$ a $\lambda$-Dirichlet group (with Haar measure $m$ ). Then the map

$$
\mathcal{R}: H_{1}^{\lambda}(G) \rightarrow M_{\lambda}(G), \quad f \mapsto f d m
$$

is an onto isometry. In particular $\mathcal{H}_{1}(\lambda)=M_{\lambda}(G)$.

Actually it was discovered in [26, Theorem 4.25] that Theorem 4.6 is a fairly simple consequence of a more general result from [33, Theorem 4]; see [26, §4.7] for a discussion on that. A proof within the theory of Dirichlet series can be found in [15].

4.4. Montel theorem. In [4, Lemma 18] Bayart proves that for every bounded sequence $\left(D^{N}\right) \subset \mathcal{D}_{\infty}((\log n))$ there is a subsequence $\left(D^{N_{k}}\right)$ and $D \in \mathcal{D}_{\infty}((\log n))$ such that $\left(D^{N_{k}}\right)$ converges uniformly to $D$ on $[R e>\varepsilon$ ] for all $\varepsilon>0$. See also [45, Theorem 6.3.1, p. 147], and [23. Theorem 3.11], where Bayart's Montel theorem is deduced from a Montel type theorem for $H_{\infty}\left(B_{c_{0}}\right)$ combined with (6) and (8). This fact extends to $\lambda$ 's satisfying Bohr's theorem (recall Corollary 4.2 as well as Theorem 2.10); the proof can be found in [28, Theorem 5.8] (and for a slightly weaker version in [26, Theorem 4.19]).

TheOREM 4.7. Let Bohr's theorem hold for $\lambda$. Then for every $1 \leq p \leq \infty$ and every bounded sequence $\left(D^{N}\right) \subset \mathcal{H}_{p}(\lambda)$ there is a subsequence $\left(D^{N_{k}}\right)$ and $D \in \mathcal{H}_{p}(\lambda)$ such that for all $\varepsilon>0$ the translations about $\varepsilon$

$$
D_{\varepsilon}^{N_{k}}=\sum a_{n}\left(D^{N_{k}}\right) e^{-\varepsilon \lambda_{n}} e^{-\lambda_{n} s}
$$

converge to $D$ in $\mathcal{H}_{p}(\lambda)$ as $k$ tends to $\infty$.

4.5. Hilbert's criterion. As already mentioned the 'ordinary' $\mathcal{D}_{\infty}$ isometrically equals $H_{\infty}\left(B_{c_{0}}\right)$, identifying Dirichlet and monomial coefficients. A crucial argument in the proof of this result (see [23, §2.3]) is that a continuous function $f: B_{c_{0}} \rightarrow \mathbb{C}$ belongs to $H_{\infty}\left(B_{c_{0}}\right)$ if and only if all its restrictions $\left.f\right|_{N}: \mathbb{D}^{N} \rightarrow \mathbb{C}$ belong to $H_{\infty}\left(\mathbb{D}^{N}\right)$ and $\sup _{N}\left\|\left.f\right|_{N}\right\|_{\infty}<\infty$.

Formulated for ordinary Dirichlet series this shows that $D=\sum a_{n} n^{-s} \in \mathcal{D}_{\infty}$ if and only if for all $N$ its so-called $N$-th abschnitte $\left.D\right|_{N}=\sum a_{n} n^{-s}$, where the sum is taken over those $n$ which only have the first $N$ primes as divisors, belong to $\mathcal{D}_{\infty}$ with uniformly bounded norms. In more vague terms, $D \in \mathcal{D}_{\infty}$ if and only if all its finite dimensional blocks are in $\mathcal{D}_{\infty}$ with uniformly bounded norms (see also [23, Theorem 3.11]).

This phenomenon also is true in the general case. Given some decomposition $\lambda=$ $(R, B)$ the $N$-th abschnitt $\left.D\right|_{N}$ of a $\lambda$-Dirichlet series $D$ is the sum $\sum a_{n}(D) e^{-\lambda_{n} s}$, where $a_{n}(D) \neq 0$ implies that $\lambda_{n}$ depends only on the first $N$ basis elements $b_{1}, \ldots, b_{N}$.

As proved in [15] and [49], general Dirichlet series $D \in \mathcal{H}_{p}(\lambda)$ for every frequency are again 'determined by their finite dimensional parts'. A weaker form of the following theorem can also be found in [26, Theorem 4.22].

Theorem 4.8. Let $1 \leq p \leq \infty$ and let $\lambda=(R, B)$ be an arbitrary frequency. Let $D$ be a formal $\lambda$-Dirichlet series. Then $D \in \mathcal{H}_{p}(\lambda)$ if and only if the $N$-th abschnitt $\left.D\right|_{N} \in \mathcal{H}_{p}(\lambda)$ for all $N \in \mathbb{N}$ and $\sup _{N}\left\|\left.D\right|_{N}\right\|_{p}<\infty$. Moreover, in this case $\|D\|_{p}=\sup _{N \in \mathbb{N}}\left\|\left.D\right|_{N}\right\|_{p}$.

By Theorem 4.1 we obtain the following particular case: If $\lambda$ satisfies Bohr's theorem, then $D \in \mathcal{D}_{\infty}(\lambda)$ if and only if $\left.D\right|_{N} \in \mathcal{D}_{\infty}(\lambda)$ for all $N \in \mathbb{N}$ and $\sup _{N}\left\|\left.D\right|_{N}\right\|_{\infty}<\infty$. 
4.6. Helson's theorem. A celebrated result of Helson [39, §2] (see also [40, Theorem 9, p. 29]) on general Dirichlet series $\sum a_{n} e^{-\lambda_{n} s}$ states that if $\lambda$ satisfies $(B C)$ and $\left(a_{n}\right)$ is 2-summable, then for almost all homomorphism $\omega:(\mathbb{R},+) \rightarrow \mathbb{T}$ the Dirichlet series $\sum a_{n} \omega\left(\lambda_{n}\right) e^{-\lambda_{n} s}$ converges on the open right halfplane $[R e>0]$, or equivalently, for all $u>0$ the series $\sum a_{n} e^{-\lambda_{n} u} h_{\lambda_{n}}$ converges almost everywhere on $\overline{\mathbb{R}}$ (note that if we for all rational $u$ 's collect zero sets in $\overline{\mathbb{R}}$, then we get the aforementioned result). See also [37, Theorem 4.4] for the ordinary case $\lambda=(\log n)$.

Using our terminology, let us rephrase this result for square summable functions on $\lambda$-Dirichlet groups: Given a frequency $\lambda$ with $(B C)$, a $\lambda$-Dirichlet group $(G, \beta)$, and some $f \in H_{2}^{\lambda}(G)$, then for every $u>0$ the series

$$
\sum \widehat{f}\left(h_{\lambda_{n}}\right) e^{-\lambda_{n} u} h_{\lambda_{n}}
$$

converges almost everywhere on $G$.

This result extends to functions in $H_{p}^{\lambda}(G)$. To see this recall that for $u>0$ the Poisson kernel

$$
P_{u}(t):=\frac{1}{\pi} \frac{u}{u^{2}+t^{2}}: \mathbb{R} \rightarrow \mathbb{R}
$$

is Lebesgue integrable with $\left\|P_{u}\right\|_{1}=1$. Hence, by the Riesz representation theorem, for every $\lambda$-Dirichlet group $(G, \beta)$ the continuous functional

$$
T_{u}: C(G) \rightarrow \mathbb{C}, \quad g \mapsto \int_{\mathbb{R}}(g \circ \beta)(t) P_{u}(t) d t
$$

defines a measure on $G$ which we call the 'Poisson measure' on $G$ denoted by $p_{u}$.

The following theorem from [28, Theorem 4.2] is a far reaching extension of Helson's theorem. It in fact shows that, given an appropriate frequency $\lambda$, Helson's theorem extends to functions $f$ from our scale $H_{p}^{\lambda}(G), 1 \leq p<\infty$, modelled on $\lambda$-Dirichlet groups $G$. Moreover, it describes the $G$-a.e. pointwise limit of the Fourier series $\sum_{n=1}^{\infty} \widehat{f}\left(h_{\lambda_{n}}\right) e^{-\lambda_{n} u} h_{\lambda_{n}}$ in terms of convolution of $f$ with the Poisson measure $p_{u}$ on $G$, and-most important-it adds the relevant maximal inequality.

THEOREM 4.9. Let $\lambda$ satisfy $(B C),(G, \beta)$ be a $\lambda$-Dirichlet group, and $f \in H_{p}^{\lambda}(G)$ with $1 \leq p \leq \infty$. Then for every $u>0$

$$
f * p_{u}=\sum_{n=1}^{\infty} \widehat{f}\left(h_{\lambda_{n}}\right) e^{-\lambda_{n} u} h_{\lambda_{n}}
$$

almost everywhere on $G$. Moreover, for every $u>0$ there is a constant $C=C(u, \lambda)$ such that for all $1 \leq p \leq \infty$ and for all $f \in H_{p}^{\lambda}(G)$

$$
\left\|\sup _{N}\left|\sum_{n=1}^{N} \widehat{f}\left(h_{\lambda_{n}}\right) e^{-\lambda_{n} u} h_{\lambda_{n}}\right|\right\|_{p} \leq C\|f\|_{p} .
$$

REMARK 4.10. The case $p=\infty$ in 26 easily recovers that frequencies with $(B C)$ satisfy Bohr's theorem, i.e. for every somewhere convergent $\lambda$-Dirichlet series $D$ we have $\sigma_{b}^{\text {ext }}(D)=\sigma_{u}(D)$. 
REMARK 4.11. We also like to mention that in [28, Theorem 3.2] we extend Theorem 4.9 to the class of $\lambda$ 's with $(L C)$, with the difference that then the constant $C$ from 26 depends on $p$ and the case $p=1$ requires to replace the $L_{1}(G)$-norm by the norm of the weak $L_{1}(G)$ space $L_{1, \infty}(G)$.

Problem 4.12. Assuming $(L C)$ for $\lambda$, is it possible to prove (26) with a constant independent of $p$ ?

Clearly, if the answer here would be affirmative, then this again would imply Bohr's theorem which is already known to be valid under $(L C)$ (see Theorem 2.10).

4.7. Vertical limits. Let us reformulate Theorem 4.9 in terms of general Dirichlet series. Given a $\lambda$-Dirichlet series $D=\sum a_{n} e^{-\lambda_{n} s}$ and a $\lambda$-Dirichlet group $(G, \beta)$, we for $\omega \in G$ call the Dirichlet series

$$
D^{\omega}=\sum a_{n} h_{\lambda_{n}}(\omega) e^{-\lambda_{n} s}
$$

a vertical limit of $D$. The following characterization of vertical limits justifies this name (see [26, Proposition 4.6]).

REMARK 4.13. Let $(G, \beta)$ be a $\lambda$-Dirichlet group, and $D=\sum a_{n} e^{-\lambda_{n} s}$ a $\lambda$-Dirichlet series with $\sigma_{a}(D) \leq 0$. Then

(1) For every $\omega \in G$ there is a sequence $\left(\tau_{k}\right) \subset \mathbb{R}$ such for all $\varepsilon>0$ the 'vertical translations'

$$
D_{i \tau_{k}}=\sum a_{n} e^{-i \lambda_{n} \tau_{k}} e^{-\lambda_{n} s}
$$

converge to $D^{\omega}$ uniformly on $[R e>\varepsilon]$.

(2) Assume conversely that the $\left(D_{i \tau_{k}}\right)$ for some $\left(\tau_{k}\right) \subset \mathbb{R}$ converge uniformly on $[R e>\varepsilon]$ to a holomorphic function $f$ for every $\varepsilon>0$. Then there is $\omega \in G$ such that $f(s)=$ $D^{\omega}(s)$ for all $s \in[R e>0]$.

Note that the notion of vertical limits allows us to rephrase Helson's theorem from the preceding section as follows: Given the $\lambda$-Dirichlet group $G=\left(\overline{\mathbb{R}}, \beta_{\overline{\mathbb{R}}}\right)$ with $(B C)$ for $\lambda$ and $D \in \mathcal{H}_{2}(\lambda)$, then $\overline{\mathbb{R}}$-almost all vertical limits $D^{\omega}$ of $D$ converge on $[R e>0]$.

The following result from [28, Theorem 3.1 and Theorem 4.1] is then a reformulation of Theorem 4.9 in terms of Dirichlet series.

TheOREm 4.14. Let $(G, \beta)$ be a $\lambda$-Dirichlet group for a frequency $\lambda$ with $(B C)$, and $D \in \mathcal{H}_{p}(\lambda)$ with $1 \leq p<\infty$. Then almost all vertical limits

$$
D^{\omega}=\sum a_{n}(D) h_{\lambda_{n}}(\omega) e^{-\lambda_{n} s}, \quad \omega \in G,
$$

converge on $[R e>0]$. Moreover, for every $u>0$ there is a constant $C=C(u, \lambda)$ such that for all $1 \leq p<\infty$, all $D \in \mathcal{H}_{p}(\lambda)$ and almost all $\omega \in G$

$$
\lim _{T \rightarrow \infty}\left(\frac{1}{2 T} \int_{-T}^{T} \sup _{N}\left|\sum_{n=1}^{N} a_{n} h_{\lambda_{n}}(\omega) e^{-(u+i t) \lambda_{n}}\right|^{p} d t\right)^{1 / p} \leq C\|D\|_{p} .
$$

Again convolution allows relating the values of the vertical limits $D^{\omega}$ of a Dirichlet series $D \in \mathcal{H}_{p}(\lambda)$ to its associated function $f \in H_{p}^{\lambda}(G)$. The vertical limits $D^{\omega}$ in some sense recover the associated function $f$ on vertical lines (see [28]). Note that, given 
$f \in L_{p}(G)$, almost all $\omega \in G$ define a measurable function $f_{\omega}(t):=f(\omega \beta(t))$, which itself is defined for almost all $t \in \mathbb{R}$. In particular, the convolution

$$
f_{\omega} * P_{u}(t):=\int_{\mathbb{R}} f_{\omega}(t-y) P_{u}(y) d y
$$

is defined almost everywhere on $\mathbb{R}$ (see [26, Lemma 3.11]). The following corollary is content of [28, Theorem 3.1].

Corollary 4.15. Let $1 \leq p<\infty, \lambda$ satisfy $(L C)$, and $(G, \beta)$ be a $\lambda$-Dirichlet group. If $f \in H_{p}^{\lambda}(G)$ and $D \in \mathcal{H}_{p}(\lambda)$ are associated to each other, then there is a null set $N \subset G$ such that for all $\omega \notin N$, for all $u>0$ and for almost all $t \in \mathbb{R}$ we have

$$
D^{\omega}(u+i t)=\left(f_{\omega} * P_{u}\right)(t) .
$$

Let us consider the particular case of ordinary Dirichlet series. We denote by $\Xi$ the set of all completely multiplicative characters $\chi: \mathbb{N} \rightarrow \mathbb{T}$ (that is $\chi(n m)=\chi(n) \chi(m)$ for all $m, n)$ which with the pointwise multiplication forms an abelian group. Looking at the group isomorphism

$$
\iota: \Xi \rightarrow \mathbb{T}^{\infty}, \quad \chi \mapsto\left(\chi\left(p_{n}\right)\right)
$$

where $\mathfrak{p}=\left(p_{n}\right)$ again denotes the sequence of prime numbers, we see that $\Xi$ also forms a compact abelian group, and its Haar measure $d \chi$ is the push-forward measure of $d z$ through $\iota^{-1}$. Then applying Theorem 4.14 to the $(\log n)$-Dirichlet group $\Xi$ we obtain the following consequence (compare also with [30, Section 3]).

COROLlary 4.16. For all $u>0$ there is a constant $C=C(u)$ such that for all $1 \leq p<\infty$ and all Dirichlet series $\sum a_{n} n^{-s} \in \mathcal{H}_{p}((\log n))$

$$
\left(\int_{\Xi} \sup _{N}\left|\sum_{n=1}^{N} a_{n} \chi(n) n^{-u}\right|^{p} d \chi\right)^{1 / p} \leq C\|D\|_{p}
$$

In particular, almost all vertical limits $D^{\chi}(s)=\sum a_{n} \chi(n) n^{-s}$ converge on $[$ Re $>0]$.

REMARK 4.17. Helson in [39, §4] deduces from this result the curious fact that Riemann's conjecture holds for almost all randomized $\zeta$-functions $\sum \chi(n) n^{-s}$, in the sense that almost all of them converge pointwise on $[R e>1 / 2]$ without having any zero on this halfplane (see also [37, Corollary 4.7]).

Bayart in [4] deduces the 'almost everywhere part' of Corollary 4.16 for the case $p=2$ from the Menchoff-Rademacher theorem on almost everywhere convergence of orthonormal series, and then he extends it to the range $1 \leq p<\infty$ using the 'hypercontractivity of the Poisson kernel'. For a brief survey on all this in the ordinary case only, we also refer to [27].

Let us explain what is meant by 'hypercontractivity of the Poisson kernel'. Recall the definition

$$
p_{r}(w):=\sum_{n=-\infty}^{\infty} r^{|n|} w^{n}, \quad 0<r<1,
$$

of the Poisson kernel on $\mathbb{T}$, and consider for all $1 \leq p<q<\infty$ the convolution operator

$$
\mathcal{P}_{r}: H_{p}(\mathbb{T}) \rightarrow H_{q}(\mathbb{T}), \quad f \mapsto f * p_{r}(w)=\sum_{n=0}^{\infty} \widehat{f}(n)(r w)^{n}
$$


An easy computation shows that $\left\|\mathcal{P}_{r}\right\| \leq \frac{1}{1-r}$ (independently of $p, q$ ), but this bound improves considerably depending on the relation of $p, q, r$. Weissler in [51] shows that $\left\|\mathcal{P}_{r}\right\|=1$ if and only if $r^{2} \leq \frac{p}{q}$; a result which is referred to as the 'hypercontractivity of the Poisson kernel'. Then using this result 'in each variable separately' shows that for all $u>0$ and $1 \leq p, q<\infty$ the translation operator

$$
\mathcal{P}_{u}: \mathcal{H}_{p} \rightarrow \mathcal{H}_{q}, \quad D \mapsto D_{u}=\sum a_{n}(D) n^{-u} n^{-s},
$$

is well-defined and bounded (see [23, Theorem 12.9] for the details on all of that). This explains, why in the ordinary case 'the almost everywhere part' of Corollary 4.16 follows in the range $1 \leq p<\infty$, once this result is proven for $p=2$. For general Dirichlet series 'hypercontractivity results' are also available assuming that $L(\lambda)<\infty$, and the following result can be found in [49].

Theorem 4.18. Let $\lambda$ be a frequency with $L(\lambda)<\infty$ and $D \in \mathcal{H}_{p}(\lambda)$, where $1 \leq p<\infty$. Then for every $u>0$ and $1 \leq q<\infty$ we have $D_{u} \in \mathcal{H}_{q}(\lambda)$. Moreover, there is a constant $C=C(\lambda, u, p, q)$ such that $\left\|D_{u}\right\|_{q} \leq C\|D\|_{p}$ for all $D \in \mathcal{H}_{p}(\lambda)$.

Hence, as a consequence 27) holds if $L(\lambda)<\infty$. Since $(L C)$ does not necessarily imply $L(\lambda)<\infty$, our proof of Theorem 4.14 in [28] has to follow an entirely different approach. Nevertheless the following problem seems interesting in itself.

Problem 4.19. Does Theorem 4.18 hold under $(L C)$ ?

From [23, §12] we know that for $1 \leq p<\infty$

$$
\sup _{D \in \mathcal{H}_{p}} \sigma_{c}(D)=\sup _{D \in \mathcal{H}_{p}} \sigma_{a}(D)=\frac{1}{2} .
$$

The second equality is implicitly proven in [4], and in view of the coefficient preserving identity $\mathcal{H}_{p}=H_{p}\left(\mathbb{T}^{\infty}\right)$, it implies that the infimum over all $u>0$ such that for each $f \in H_{p}\left(\mathbb{T}^{\infty}\right)$ the series

$$
\sum_{\alpha} \widehat{f}(\alpha)\left(\frac{z}{\mathfrak{p}^{u}}\right)^{\alpha}
$$

converges (here we of course talk about absolutely convergence), equals $1 / 2$ (compare also with (10)). The next result from [28] shows that there is no such 'wall' if we order the sum 'blockwise according to the order of $\mathbb{N}$ '.

Corollary 4.20. Let $1 \leq p<\infty$ and $f \in H_{p}\left(\mathbb{T}^{\infty}\right)$. Then for all $u>0$

$$
\lim _{N \rightarrow \infty} \sum_{\mathfrak{p}^{\alpha} \leq N} \widehat{f}(\alpha)\left(\frac{z}{\mathfrak{p}^{u}}\right)^{\alpha} \quad \text { a.e. on } \mathbb{T}^{\infty},
$$

and moreover

$$
\left(\int_{\mathbb{T}^{\infty}} \sup _{\sigma \geq u} \sup _{N}\left|\sum_{\mathfrak{p}^{\alpha} \leq N} c_{\alpha}\left(\frac{z}{\mathfrak{p}^{\sigma}}\right)^{\alpha}\right|^{p} d z\right)^{1 / p} \leq C\|f\|_{p},
$$

where $C=C(u)$ only depends on $u$.

What about the case $\omega=1$ (the neutral element of $G$ ) in Theorem 4.14 i.e. pointwise convergence of Dirichlet series from $\mathcal{H}_{p}(\lambda)$ ? Another consequence of Theorem 4.18 shows 
that for every frequency $\lambda$ we have

$$
\sup _{D \in \mathcal{H}_{p}(\lambda)} \sigma_{a}(D) \leq \frac{L(\lambda)}{2} .
$$

In the particular case $\lambda=(\log n)$, we know from 29 that this upper bound is attained, that is

$$
\sup _{D \in \mathcal{H}_{p}} \sigma_{c}(D)=\sup _{D \in \mathcal{H}_{p}} \sigma_{a}(D)=\frac{L((\log n))}{2} .
$$

Problem 4.21. Let $\lambda$ be a frequency. Give reasonable upper and lower estimates for the quantities $\sup _{D \in \mathcal{H}_{p}(\lambda)} \sigma_{c}(D)$ and $\sup _{D \in \mathcal{H}_{p}(\lambda)} \sigma_{a}(D)$, for example in terms of $L(\lambda)$.

Moreover, Hedenmalm and Saksman in [38] prove that every Dirichlet series $D \in \mathcal{H}_{2}$ converges almost everywhere on $\left[R e=\frac{1}{2}\right]$.

Problem 4.22. Let $1 \leq p<2$. Does every $D \in \mathcal{H}_{p}$ converge almost everywhere on the vertical line $\left[R e=\frac{1}{2}\right]$ ?

Problem 4.23. Assume that $L(\lambda)<\infty$. Does every $D \in \mathcal{H}_{2}(\lambda)$ converge almost everywhere on $\left[R e=\frac{L(\lambda)}{2}\right]$ ?

4.8. Carleson's theorem. What happens if we consider the case $u=0$ in the preceding two sections, in particular in Theorem 4.9?

It turns out that this question is intimately related with one of the most famous theorems in Fourier analysis, namely Carleson's theorem: The Fourier series of every $f \in L_{2}(\mathbb{T})$ converges almost everywhere. More generally, the Carleson-Hunt theorem states that if $f \in L_{p}(\mathbb{T}), 1<p<\infty$, then $f(z)=\sum_{n=1}^{\infty} \widehat{f}(n) z^{n}$ for almost all $z \in \mathbb{T}$ and

$$
\left\|\sup _{N}\left|\sum_{n=-N}^{N} \widehat{f}(n) z^{n}\right|\right\|_{p} \leq C\|f\|_{p} .
$$

The following theorem extends the Carleson-Hunt theorem to our setting of $\lambda$-Dirichlet groups, and this means a strong improvement of Theorem 4.4 .

THEOREM 4.24. Let $\lambda$ be a frequency, $(G, \beta)$ a $\lambda$-Dirichlet group, and $1<p<\infty$. Then for every $f \in H_{p}^{\lambda}(G)$

$$
f=\sum_{n=1}^{\infty} \widehat{f}\left(h_{\lambda_{n}}\right) h_{\lambda_{n}}
$$

almost everywhere on $G$, and

$$
\left\|\sup _{N}\left|\sum_{n=1}^{N} \widehat{f}\left(h_{\lambda_{n}}\right) h_{\lambda_{n}}\right|\right\|_{p} \leq C\|f\|_{p},
$$

where $C=C(p)$ is a constant which only depends on $p$.

For ordinary Dirichlet series the case $p=2$ is due to [38, Theorem 1.4], whereas the more general case given here is a reformulation of a result of [34, Theorem 13] in terms of $\lambda$-Dirichlet groups. The proof given in [28, Theorem 2.2] follows closely the ideas of 38] combining them with the Carleson-Hunt theorem and a magic trick of Fefferman from [35]. 
The following interesting consequence for $1<p<\infty$ improves (the first part of) Theorem 4.14 (see again [28, Theorem 2.1]).

Corollary 4.25. Let $\lambda$ be a frequency and $D \in \mathcal{H}_{p}(\lambda), 1<p<\infty$. Then for any $\lambda$-Dirichlet group $(G, \beta)$ almost all vertical limits $D^{\omega}$ converge almost everywhere on the imaginary axis $[R e=0]$.

In view of Theorem 4.9 and Theorem 4.14 (recall also Remark 4.11) it is somewhat surprising that Theorem 4.24 and its Corollary 4.25 do not require $(L C)$ for $\lambda$.

PROBlEm 4.26. Do Theorems 4.9 and 4.14 hold for a wider class of frequencies than the class of $\lambda$ 's satisfying $(L C)$ ? Is this even true without any condition on $\lambda$ ?

Theorem 4.24 easily translates into a sort of Carleson-Hunt theorem for functions on the polytorus $\mathbb{T}^{\infty}$. Obviously, given some $f \in H_{p}\left(\mathbb{T}^{\infty}\right)$ with $1<p<\infty$, there in general is no $z \in \mathbb{T}^{\infty}$ such that $f(z)=\sum_{\alpha} \widehat{f}(\alpha) z^{\alpha}$ (since here absolute convergence is required).

Corollary 4.27. Let $1<p<\infty$ and $f \in H_{p}\left(\mathbb{T}^{\infty}\right)$. Then for almost all $z \in \mathbb{T}^{\infty}$

$$
f(z)=\lim _{N \rightarrow \infty} \sum_{\mathfrak{p}^{\alpha} \leq N} \widehat{f}(\alpha) z^{\alpha},
$$

and moreover,

$$
\left\|\sup _{N}\left|\sum_{\mathfrak{p}^{\alpha} \leq N} \widehat{f}(\alpha) z^{\alpha}\right|\right\|_{p} \leq C(p)\|f\|_{p} .
$$

REMARK 4.28. Since the Carleson-Hunt theorem fails in $H_{1}(\mathbb{T})$, Corollary 4.27 also fails for $p=1$. We like to mention that the typical Riesz means from 18 may up to some point compensate this loss. In [29] we give a careful discussion of all this, and prove among others that, if $f \in H_{1}\left(\mathbb{T}^{\infty}\right)$, then for every $k>0$

$$
f(z)=\lim _{x \rightarrow \infty} \sum_{\log \left(\mathfrak{p}^{\alpha}\right)<x} \widehat{f}(\alpha)\left(1-\frac{\log \left(\mathfrak{p}^{\alpha}\right)}{x}\right)^{k} z^{\alpha},
$$

almost everywhere on $\mathbb{T}^{\infty}$.

5. Vector-valued aspects of general Dirichlet series. We now briefly discuss some results on vector-valued general Dirichlet series $\sum a_{n} e^{-\lambda_{n} s}$, where $\left(a_{n}\right) \subset X$ for some Banach space $X$. All results are related to the above topics on general Dirichlet series with scalar coefficients, and most of them are taken from [15].

In the ordinary case $\lambda=(\log n)$ the study of functional analytic aspects of vectorvalued Dirichlet series accumulated quite some amount of research, see e.g. [15], 16], [17], [18], 19], 222, 25], 30], or [31. As an example we recall the following strong extension of (2) from 22]: The maximal width of the strip of uniform, non-absolute convergence of $X$-valued ordinary Dirichlet series is given by the formula

$$
S(X)=1-\frac{1}{\cot (X)},
$$

where $\cot (X)$ denotes the infimum over all $2 \leq q \leq \infty$ for which $X$ has cotype $q$ (see also [23, Theorem 26.4]). 
Given a Banach space $X$ and a frequency $\lambda$, we denote by $\mathcal{D}(\lambda, X)$ the space of all $\lambda$-Dirichlet series with coefficients in $X$, and by $\mathcal{D}_{\infty}(\lambda, X)$ the space of all $D \in \mathcal{D}(\lambda, X)$ which on $[R e>0]$ define bounded (and then necessarily holomorphic) functions. Moreover, the by now obvious definition of the a priori larger space $\mathcal{D}_{\infty}^{\text {ext }}(\lambda, X)$ is then clear.

The Hahn-Banach theorem transports many results from scalar-valued Dirichlet series to vector-valued ones. For instance this way we may easily deduce from Corollary 2.7 that $\left(\mathcal{D}_{\infty}(\lambda, X),\|\cdot\|_{\infty}\right)$ is a normed space with

$$
\sup _{n \in \mathbb{N}}\left\|a_{n}\right\|_{X} \leq\|D\|_{\infty} \quad \text { for all } D \in \mathcal{D}_{\infty}(\lambda, X) .
$$

In [15] it is shown that $\mathcal{D}_{\infty}(\lambda)$ is complete if and only if $\mathcal{D}_{\infty}(\lambda, X)$ is for every non-trivial Banach space $X$.

Another straightforward application of the Hahn-Banach theorem yields that the vector-valued version of Theorem 2.4 holds, and consequently also the qualitative versions (16) and (17) of Bohr's theorem (where in all inequalities the absolute value of course has to be replaced by the norm of $X$ ).

Given $1 \leq p \leq \infty$, a Banach space $X$ and a frequency $\lambda$, the definition of Hardy spaces of $X$-valued $\lambda$-Dirichlet series extends naturally to the vector-valued situation. As in the scalar case we for any $\lambda$-Dirichlet group $(G, \beta)$ define the Banach space

$$
H_{p}^{\lambda}(G, X):=\left\{f \in L_{p}(G, X) \mid f: \widehat{G} \rightarrow X \text { is supported on all } h_{\lambda_{n}}, n \in \mathbb{N}\right\},
$$

and consider the vector-valued Bohr map

$$
\mathcal{B}: H_{p}^{\lambda}(G, X) \hookrightarrow \mathcal{D}(\lambda, X), \quad f \mapsto D=\sum \widehat{f}\left(h_{\lambda_{n}}\right) e^{-\lambda_{n} s} .
$$

Then it again turns out that the Hardy space

$$
\mathcal{H}_{p}(\lambda, X):=\mathcal{B}\left(H_{p}^{\lambda}(G, X)\right)
$$

of all $X$-valued $\lambda$-Dirichlet series together with the norm $\|D\|_{p}:=\|f\|_{p}$, forms a Banach space which is independent of the chosen $\lambda$-Dirichlet group.

In this final section of our survey we focus on two different topics from [15]. In the first one we ask up to which extension Theorem 4.1 and Corollary 4.2 transfer to the vectorvalued setting. More precisely, for which Banach spaces $X$ and frequencies $\lambda$ does the equality $\mathcal{D}_{\infty}(\lambda, X)=\mathcal{H}_{\infty}(\lambda, X)$ hold (with an coefficient preserving isometry). Secondly, we revisit the maximal inequalities from Sections 4.6 and 4.8 in the vector-valued case.

5.1. Coincidence of vector-valued $H_{\infty}$ 's. We recall that a Banach space $X$ has the analytic Radon-Nikodym property (short ARNP) if every $f \in H_{\infty}(\mathbb{D}, X)$ has radial limits in almost all $w \in \mathbb{T}$. This happens if and only if $H_{\infty}(\mathbb{T}, X)=H_{\infty}(\mathbb{D}, X)$ (via an isometry identifying Fourier and monomial coefficients). Then it is straightforward to see that $\mathcal{D}_{\infty}((n), X)=\mathcal{H}_{\infty}((n), X)$ if and only if $X$ has ARNP.

The results of [24, Theorem 5.5 and Theorem 6.2] (see also [23, Theorem 24.17]) show that this result also holds for ordinary Dirichlet series, and in the following theorem we state an extension of all this for general Dirichlet series which is proven in [15]. 
TheOREM 5.1. Let $\lambda$ be any frequency and $X$ a non-trivial Banach space with ARNP. Then $\mathcal{D}_{\infty}(\lambda)=\mathcal{H}_{\infty}(\lambda)$ if and only if

$$
\mathcal{D}_{\infty}(\lambda, X)=\mathcal{H}_{\infty}(\lambda, X) .
$$

In particular, 30 holds whenever $X$ has ARNP and $\lambda$ satisfies Bohr's theorem.

But in fact there are Banach spaces $X$ without ARNP as well as frequencies $\lambda$ for which $\mathcal{D}_{\infty}(\lambda)=\mathcal{H}_{\infty}(\lambda)$, such that $(30)$ is no equality. To see this we extend Theorem 2.9 to the vector-valued case. Denote by $\ell_{1}^{w}(X)$ the Banach space of all weakly summable $X$-valued sequences $\left(a_{n}\right)$ with the norm

$$
w\left(\left(a_{n}\right)\right)=\sup _{x^{\prime} \in B_{X^{\prime}}} \sum_{n=1}^{\infty}\left|x^{\prime}\left(a_{n}\right)\right|
$$

and by $\ell_{1}^{w, 0}(X)$ the closed subspace of $\ell_{1}^{w}(X)$ consisting of all sequences $\left(a_{n}\right)$ which are unconditionally summable. Recall that $\ell_{1}^{w, 0}(X)=\ell_{1}^{w}(X)$ if and only if $X$ does not contain an isomorphic copy of $c_{0}$ (a result of Bessaga and Pełczyński, see e.g. [32, p. 45]). The following theorem is the announced extension of Theorem 2.9 from [15].

Theorem 5.2. If $\lambda$ is $\mathbb{Z}$-linearly independent, then for every Banach space $X$ the following isometric inclusions hold:

$$
\ell_{1}^{w, 0}(X) \subset \mathcal{H}_{\infty}(\lambda, X) \subset \mathcal{D}_{\infty}(\lambda, X) \subset \ell_{1}^{w}(X) .
$$

In particular, $\mathcal{D}_{\infty}(\lambda, X)=\mathcal{H}_{\infty}(\lambda, X)$ holds isometrically whenever $c_{0}$ is no isomorphic subspace of $X$.

Banach lattices have ARNP if and only if they do not contain an isomorphic copy of $c_{0}$. But in contrast to this, there are Banach spaces which fail to have ARNP and fail to contain an isomorphic copy of $c_{0}$ (see [14, $\left.\S 4\right]$ ). Consequently, Theorem 5.2 shows that if $\lambda$ is $\mathbb{Z}$-linearly independent, then the equality $\mathcal{H}_{\infty}(\lambda, X)=\mathcal{D}_{\infty}(\lambda, X)$ does not necessarily imply that $X$ has ARNP.

5.2. Maximal inequalities - vector-valued. Now we discuss the inequalities from Sections 4.2, 4.6 and 4.8 Recall that our proof of Theorem 4.4 relies explicitly on the boundedness of the Riesz projection on $L_{p}(G)$, where $G$ is some Dirichlet group. This fact remains valid for $X$-valued functions whenever $X$ is a so-called UMD-space, which, as observed in [15], is an immediate consequence of results from [2].

Theorem 5.3. Let $(G, \beta)$ be a Dirichlet group, $X$ a Banach space and $1<p<\infty$. Then $X$ has $U M D$ if and only if the Riesz projection

$$
R: L_{p}(G, X) \rightarrow L_{p}(G, X), \quad f \mapsto \sum_{x \geq 0} \widehat{f}\left(h_{x}\right) h_{x}
$$

is bounded.

As done in [26, Section 4.4] for the scalar case, a standard argument leads to the following consequence generalizing Theorem 4.4 (see once again [15]). 
Corollary 5.4. For every frequency $\lambda$ and every $U M D$-space $X$ we for $1<p<\infty$ have

$$
\sup _{N}\left\|S_{N}^{p, X}: \mathcal{H}_{p}(\lambda, X) \rightarrow \mathcal{H}_{p}(\lambda, X), \sum a_{n} e^{-\lambda_{n} s} \mapsto \sum_{n \leq N} a_{n} e^{-\lambda_{n} s}\right\|<\infty .
$$

Let us finally again turn to the Carleson-Hunt theorem. The proof of Theorem 4.24 given in [28, Theorem 2.2], extends in the following sense from scalar to vector-valued functions.

THEOREM 5.5. Assume that $X$ is a Banach space for which the Carleson-Hunt maximal inequality holds, i.e. for every $1<p<\infty$ there is some $C=C(p)>0$ such that for every $f \in L_{p}(\mathbb{T}, X)$

$$
\left\|\sup _{N}\right\| \sum_{n=-N}^{N} \widehat{f}(n) z^{n}\|\|_{L_{p}(\mathbb{T})} \leq C\|f\|_{L_{p}(\mathbb{T}, X)} .
$$

Then for every frequency $\lambda$, every $\lambda$-Dirichlet group $(G, \beta)$, and every $1<p<\infty$ we for every $f \in H_{p}^{\lambda}(G, X)$ have

$$
f=\sum_{n=1}^{\infty} \widehat{f}\left(h_{\lambda_{n}}\right) h_{\lambda_{n}}
$$

almost everywhere on $G$, and

$$
\left\|\sup _{N}\right\| \sum_{n=1}^{N} \widehat{f}\left(h_{\lambda_{n}}\right) h_{\lambda_{n}}\|\|_{p} \leq C\|f\|_{p}
$$

In particular, if $X$ is a Banach lattice with UMD, then (31) is true (see e.g. [46]), hence in this case its $X$-valued variant $[32$ is valid. Actually, it is proven in [15] that for $\mathbb{Z}$-linearly independent frequencies $\lambda$ the assumption UMD on $X$ in fact is superfluous.

Proposition 5.6. If $\lambda$ is $\mathbb{Z}$-linearly independent, then 32 holds for all Banach spaces.

To finish, we mention that 25 and the variant of 26 in Theorem 4.9 under $(L C)$ (see Remark 4.11) extend to the vector-valued case, whereas in contrast to this, our proof of the fact that under $(B C)$ the constant $C$ from 26 may be chosen independently of $p$ uses tools like the Hausdorff-Young inequality and so does not extend to the vector-valued case.

\section{References}

[1] A. Aleman, J. F. Olsen, E. Saksman, Fourier multipliers for Hardy spaces of Dirichlet series, Int. Math. Res. Not. IMRN 16 (2014), 4368-4378.

[2] N. H. Asmar, B. P. Kelly, S. Montgomery-Smith, A note on UMD spaces and transference in vector-valued function spaces, Proc. Edinburgh Math. Soc. 39 (1996), 485-490.

[3] R. Balasubramanian, B. Calado, H. Queffélec, The Bohr inequality for ordinary Dirichlet series, Studia Math. 175 (2006), 285-304.

[4] F. Bayart, Hardy spaces of Dirichlet series and their composition operators, Monatsh. Math. 136 (2002), 203-236. 
[5] F. Bayart, A. Defant, L. Frerick, M. Maestre, P. Sevilla-Peris, Multipliers of Dirichlet series and monomial series expansion of holomorphic functions in infinitely many variables, Math. Ann. 368 (2017), 837-876.

[6] A. S. Besicovitch, Almost Periodic Functions, Dover Publ., New York, 1955.

[7] H. F. Bohnenblust, E. Hille, On the absolute convergence of Dirichlet series, Ann. of Math. (2) 32 (1931), 600-622.

[8] H. Bohr, Über die gleichmäßige Konvergenz Dirichletscher Reihen, J. Reine Angew. Math. 143 (1913), 203-211.

[9] H. Bohr, Über die Bedeutung der Potenzreihen unendlich vieler Variabeln in der Theorie der Dirichletschen Reihen $\sum \frac{a_{n}}{n^{s}}$, Nachr. Ges. Wiss. Gött. Math. Phys. Kl. 4 (1913), 441-488.

[10] H. Bohr, Einige Bemerkungen über das Konvergenzproblem Dirichletscher Reihen, Rendiconti Palermo 37 (1913), 1-16.

[11] H. Bohr, Lösung des absoluten Konvergenzproblems einer allgemeinen Klasse Dirichletscher Reihen, Acta Math. 36 (1913), 197-240.

[12] H. Bohr, Zur Theorie der fastperiodischen Funktionen II, Acta Math. 46 (1925), 101-214.

[13] H. Bohr, En bemærkning om Dirichletske rakkers ligelige konvergens, Mat. Tidsskr. B (1951), 1-8.

[14] A. V. Bukhvalov, A. A. Danilevich, Boundary properties of analytic and harmonic functions with values in Banach spaces, Mat. Zametki 31 (1982), 203-214; English transl.: Math. Notes 31 (1982), 104-110.

[15] D. Carando, A. Defant, F. Marceca, I. Schoolmann, Vector-valued general Dirichlet series, in preparation.

[16] D. Carando, A. Defant, P. Sevilla-Peris, Bohr's absolute convergence problem for $\mathcal{H}_{p}$-Dirichlet series in Banach spaces, Anal. PDE 7 (2014), 513-527.

[17] D. Carando, A. Defant, P. Sevilla-Peris, Almost sure-sign convergence of Hardy-type Dirichlet series, J. Anal. Math. 135 (2018), 225-247.

[18] D. Carando, F. Marceca, M. Scotti, P. Tradacette, Random unconditional convergence of vector-valued Dirichlet series, J. Funct. Anal. 277 (2019), 3156-3178.

[19] J. Castillo-Medina, D. García, M. Maestre, Isometries between spaces of multiple Dirichlet series, J. Math. Anal. Appl. 472 (2019), 526-545.

[20] Y. S. Choi, U. Y. Kim, M. Maestre, Banach spaces of general Dirichlet series, J. Math. Anal. Appl. 465 (2018), 839-856.

[21] B. J. Cole, T. W. Gamelin, Representing measures and Hardy spaces for the infinite polydisk algebra, Proc. London Math. Soc. (3) 53 (1986), 112-142.

[22] A. Defant, D. García, M. Maestre, D. Pérez-García, Bohr's strip for vector-valued Dirichlet series, Math. Ann. 342 (2008), 533-555.

[23] A. Defant, D. García, M. Maestre, P. Sevilla-Peris, Dirichlet Series and Holomorphic Functions in High Dimensions, New Math. Monogr. 37, Cambridge Univ. Press, 2019.

[24] A. Defant, A. Pérez, Hardy spaces of vector-valued Dirichlet series, Studia Math. 243 (2018), $53-78$.

[25] A. Defant, D. Popa, U. Schwarting, Coordinatewise multiple summing operators in Banach spaces, J. Funct. Anal. 259 (2010), 220-242.

[26] A. Defant, I. Schoolmann, $\mathcal{H}_{p}$-theory of general Dirichlet series, J. Fourier Anal. Appl. 25 (2019), 3220-3258.

[27] A. Defant, I. Schoolmann, Henry Helson meets other big shots - a brief survey, Math. Proc. R. Ir. Acad. 119A (2019), 111-132. 
[28] A. Defant, I. Schoolmann, Variants of a theorem of Helson for general Dirichlet series, in preparation, 2019.

[29] A. Defant, I. Schoolmann, Riesz means in Hardy spaces on Dirichlet groups, in preparation, 2019.

[30] A. Defant, U. Schwarting, P. Sevilla-Peris, Estimates for vector-valued Dirichlet polynomials, Monatsh. Math. 175 (2014), 89-116.

[31] A. Defant, P. Sevilla-Peris, Convergence of Dirichlet polynomials in Banach spaces, Trans. Amer. Math. Soc. 363 (2011), 681-697.

[32] J. Diestel, Sequences and series in Banach spaces, Grad. Texts in Math. 92, Springer, New York, 1984.

[33] R. Doss, On the Fourier-Stieltjes transforms of singular or absolutely continuous measures, Math. Z. 97 (1967), 77-84.

[34] T. K. Duy, On convergence of Fourier series of Besicovitch almost periodic functions, Lith. Math. J. 53 (2013), 264-279.

[35] C. Fefferman, On the convergence of multiple Fourier series, Bull. Amer. Math. Soc. 77 (1971), 744-745.

[36] G. H. Hardy, M. Riesz, The general theory of Dirichlet series, Cambridge Tracts in Math. and Math. Phys. 18, Cambridge Univ. Press, Cambridge, 1915.

[37] H. Hedenmalm, P. Lindqvist, K. Seip, A Hilbert space of Dirichlet series and systems of dilated function in $L^{2}(0,1)$, Duke Math. J. 86 (1997), 1-37.

[38] H. Hedenmalm, E. Saksman, Carleson's convergence theorem for Dirichlet series, Pacific J. Math. 208 (2003), 85-109.

[39] H. Helson, Compact groups and Dirichlet series, Ark. Mat. 8 (1969), 139-143.

[40] H. Helson, Dirichlet series, H. Helson, Berkeley, CA, 2005.

[41] H. Helson, D. Lowdenslager, Prediction theory and Fourier series in several variables, Acta Math. 99 (1958), 165-202.

[42] E. Landau, Über die gleichmäßige Konvergenz Dirichletscher Reihen, Math. Z. 11 (1921), 317-318.

[43] L. Neder, Zum Konvergenzproblem der Dirichletschen Reihen beschränkter Funktionen, Math. Z. 14 (1922), 149-158.

[44] O. Perron, Zur Theorie der Dirichletschen Reihen, J. Reine Angew. Math. 134 (1908), 95-143.

[45] H. Queffélec, M. Queffélec, Diophantine Approximation and Dirichlet Series, HRI Lect. Notes 2, Hindustan Book Agency, New Delhi, 2013.

[46] J. L. Rubio de Francia, Martingale and integral transforms of Banach space valued functions, in: Probability and Banach Spaces (Zaragoza, 1985), Lecture Notes in Math. 1221, Springer, Berlin, 1986, 195-222.

[47] W. Rudin, Fourier Analysis on Groups, Interscience Tracts in Pure and Appl. Math. 12, Interscience, New York, 1962.

[48] I. Schoolmann, On Bohr's theorem for general Dirichlet series, Math. Nachr., to appear.

[49] I. Schoolmann, Ph.D. thesis, in preparation.

[50] G. Valiron, Théorie générale des séries de Dirichlet, Mémorial des Sciences Mathématiques $17,1926$.

[51] F. B. Weissler, Logarithmic Sobolev inequalities and hypercontractive estimates on the circle, J. Functional Analysis 37 (1980), 218-234. 
